Portland State University

PDXScholar

Spring 7-12-2019

\title{
Are We Providing Preferred Floral Resources for Bees in Our Neighborhoods? : Assessing the Relationship Between Small Scale Vegetation Metrics and Bee Presence in SE Portland
}

Hailey Wallace

Portland State University

Follow this and additional works at: https://pdxscholar.library.pdx.edu/open_access_etds

Part of the Environmental Sciences Commons

Let us know how access to this document benefits you.

\section{Recommended Citation}

Wallace, Hailey, "Are We Providing Preferred Floral Resources for Bees in Our Neighborhoods? : Assessing the Relationship Between Small Scale Vegetation Metrics and Bee Presence in SE Portland" (2019). Dissertations and Theses. Paper 5126.

https://doi.org/10.15760/etd.7005

This Thesis is brought to you for free and open access. It has been accepted for inclusion in Dissertations and Theses by an authorized administrator of PDXScholar. Please contact us if we can make this document more accessible: pdxscholar@pdx.edu. 
Are We Providing Preferred Floral Resources for Bees in Our Neighborhoods?:

Assessing the Relationship Between Small Scale Vegetation Metrics and Bee Presence in

SE Portland

by

Hailey Wallace

A thesis submitted in partial fulfillment of the requirements for the degree of

Master of Science

in

Environmental Science and Management

Thesis Committee:

Marion Dresner, Chair

Susan Masta

Jeffery Gerwing

Portland State University

2019 


\begin{abstract}
Bee pollinators can thrive in highly urbanized environments if their preferred floral resources and habitat types are available. Enhanced pollinator habitats are being created globally, with a large local effort in Portland, Oregon. This project determined if we were providing the most preferred floral resources at enhanced pollinator sites for bees, if floral resources were available throughout the season, and if differences in dietary preferences between native and honey bees would allow for the identification of "native bee floral resources" in South East Portland. Bee pollinators were monitored from June to August at three enhanced pollinator sites in South East Portland, Oregon. A total of 566 individual bees were observed, tiny dark bees and bumblebees composed the large majority of the urban bee composition. Vegetation metrics and bee presence were correlated using a Generalized Linear Mixed Model and significant variables that predicted bee presence included Solidago canadenisis (p-value 0.0024), density of floral resources (p-value <.001), the months June (p-value 0.02) and July (p-value 0.001$)$ as well as tiny dark bees (p-value 0.0023). Floral resource composition shifted between sites and months, with all sites having drastically reduced floral richness in August indicating diverse floral resources were not available throughout the season. Additionally, it was found that honey bees and native bees had little dietary overlap, Honey bees preferred Penstemon species and native bee preferred Solidago canadenisis. Recommendations for managing enhanced pollinator sites from this work included planting more late season plants, specific pollinator plant lists for native bees and honey bees and scheduling regular site care and watering at enhanced sites.
\end{abstract}




\section{Acknowledgements}

There are many individuals who have had influence on this project, who I would like to thank. I would like to give the biggest acknowledgement to the community science members that participated in this monitoring project. With their involvement we were able to observe 566 individual bees during our season. I was moved to see the volunteer's willingness to be involved in this project and excitement to learn more about the bees in their local area. I would also like to thank my lab partner, Michael Vermenulen for his advice and thoughtful comments in the preparation and finalization of this thesis project. I would also like to thank my advisor, Dr. Marion Dresner for her helpful and insightful comments as well as my other committee members Dr. Jeffery Gerwing and Dr. Susan Masta. I would like to thank the Edward D. and Olive C. Bushby scholarship for providing funds to host my community science workshops. I would like to thank my mom, dad and sister for their continued support during my schooling as well as reading all my thesis drafts. Lastly, I'd like to thank my partner, Pete (and dog, Mia) who provided relaxing weekend trips to the beach or forest during the stressful times of this process, read and re-read all my research related documents and who supported me wholly throughout this process. 


\section{Table of Contents}

Abstract

Acknowledgements.................................................................................................ii

List of Tables ...........................................................................................................................................v

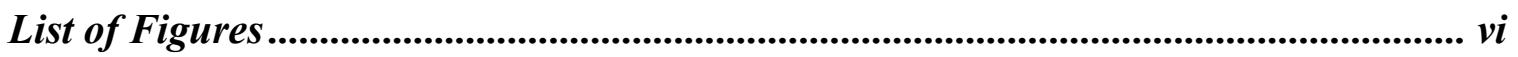

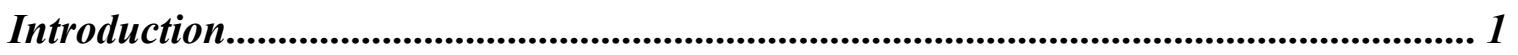

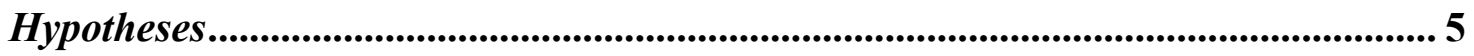

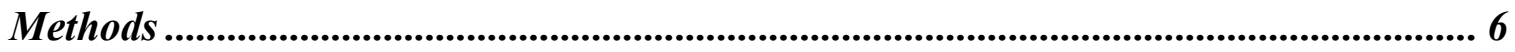

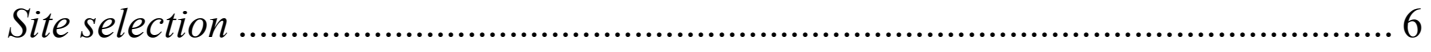

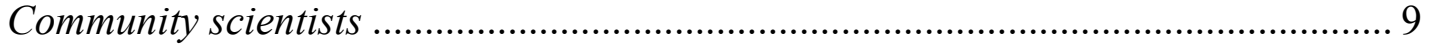

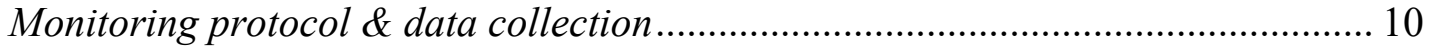

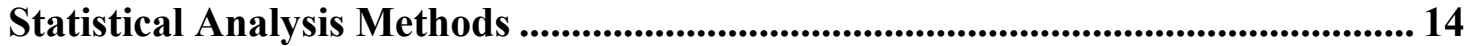

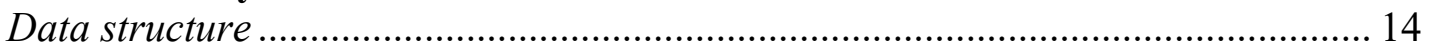

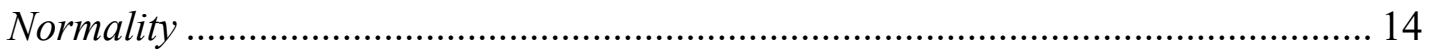

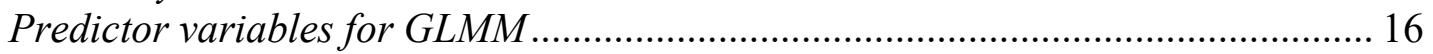

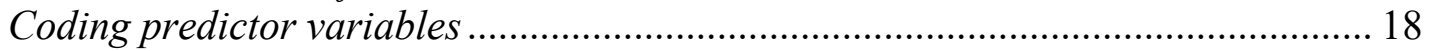

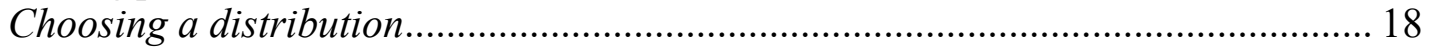

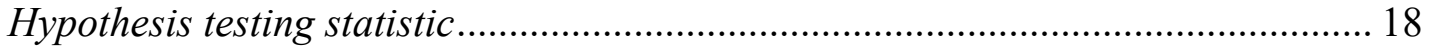

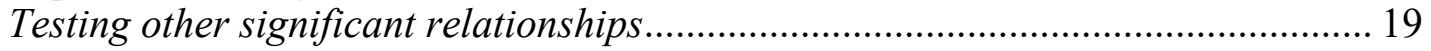

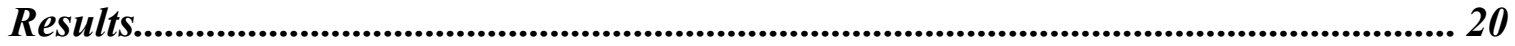

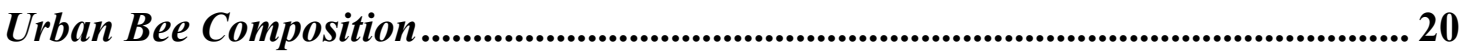

Floral Resource Composition .................................................................................. 25

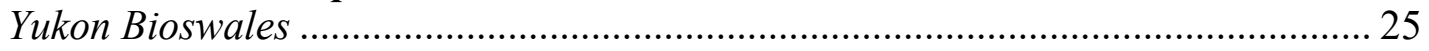

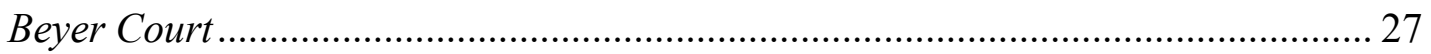

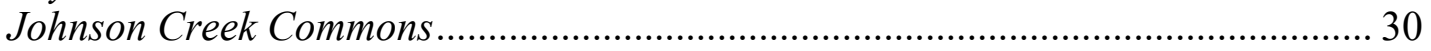

Native Bee Floral Resource Visitation ........................................................... 32

Honey Bee Floral Resource Visitation ................................................................ 32

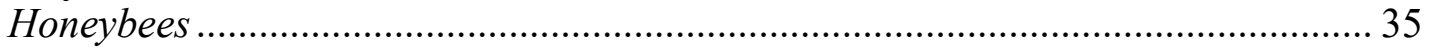

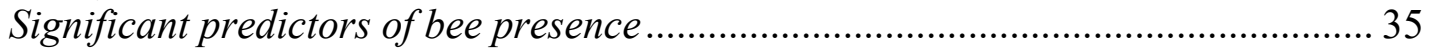

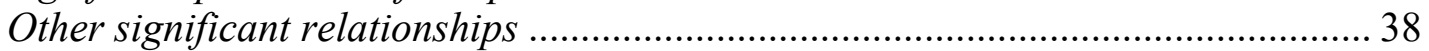

Discussion..................................................................................................................................... 40

Urban Bee Composition ....................................................................................... 40

Floral Resource Composition ............................................................................ 41

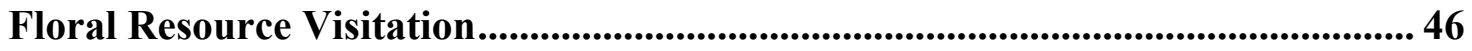

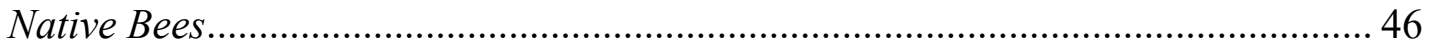

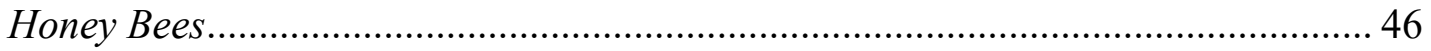

What predicted bee presence at sites in South East Portland? ............................. 47

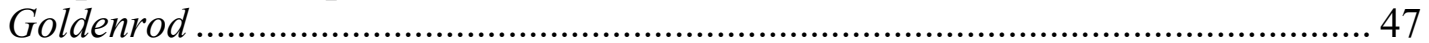

Habitat composition .................................................................................... 48 
Implications to management .......................................................................................... 49

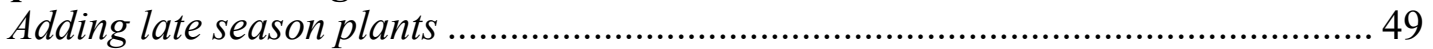

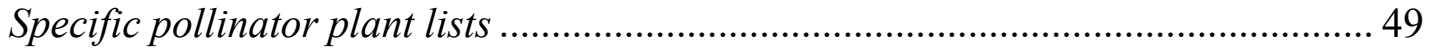

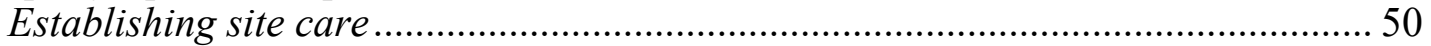

Community Science ........................................................................................ 51

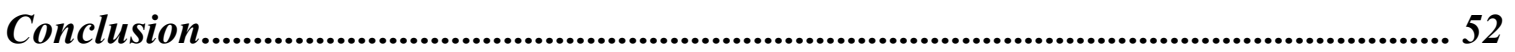

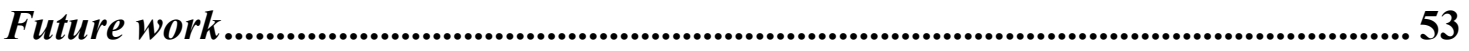

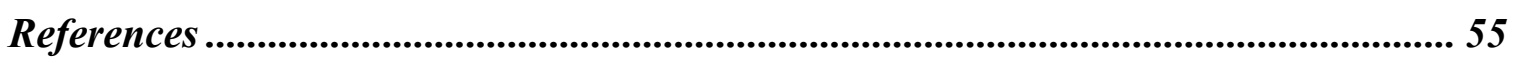

Appendices.............................................................................................................................59

Appendix A. List of plants that were initially planted by Green Lents at Johnson Creek Commons, Beyer Court Rain Garden and Yukon Bioswale enhanced pollinator sites.

Appendix B. Example of plant identification list that were provided to community scientists for monitoring bee pollinators floral associations. Appendix C. Data collection sheet for bee observational data. Data collection sheet form from Minerath et al., (2014). Appendix D. Comma-separated values (CSV) spreadsheet titled "Vegetation Survey Data".

Appendix E. Comma-separated values (CSV) spreadsheet titled "Bee Observations and Floral Resources". 
List of Tables

Table 1. Predictor variables used for predicting bee presence at sites using a GLMM model. Variables were broken into three main sections, floral resources at all sites, floral resource metrics that were measured once a month per site, and morphospecies bee group.

Table 2. Table displays numbers of bee observations of different bee groups from the entire data collection season (June, July, August). Johnson Creek Commons (JCC), Beyer Court (BC) and Yukon Bioswales (YBIO).

Table 3. Floral species richness measured by number of species flowering during vegetation surveys at Beyer Court (BC), Johnson Creek Commons (JCC) and Yukon Bioswales (YBIO) for the monitoring season June, July and August. .................... 25

Table 4. Number of honeybees observed throughout the monitoring season of June, July and August at Beyer Court (BC), Johnson Creek Commons (JCC) and Yukon Bioswales (YBIO).

Table 5. Significant variables that predicted bee presence at sites included Goldenrod ( $p$ value 0.024$)$, density of floral resources (p-value $3 \times 10-6)$, the months June (p-value 0.02 ) and July (p-value 0.001) as well as tiny dark bees (p-value 0.0023).

Significance is illustrated by asterisks. 


\section{List of Figures}

Figure 1. Map of monitoring locations in Lents, Oregon. Yukon Bioswales (3) are indicated by orange stars. Beyer Court Rain Garden indicated by the green star. Location of Johnson Creek Commons indicated by the red star.

Figure 2. Yukon Bioswales monitoring site in Lents, OR. Photo taken in May prior to

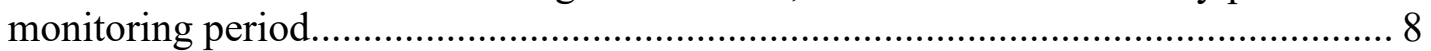

Figure 3. Beyer Court Rain Garden monitoring site in Lents, OR. Photo taken in May

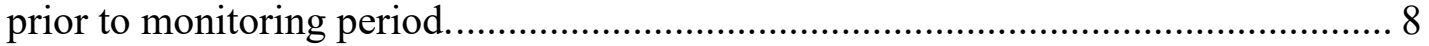

Figure 4. Johnson Creek Commons monitoring site in Lents, OR. Photo taken in May

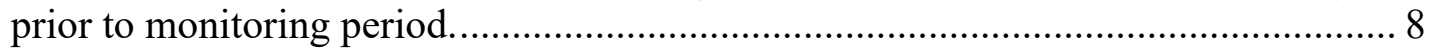

Figure 5. Transect for monitoring at Beyer Court Rain Garden................................... 12

Figure 6. Transects (outer and inner) for bee monitoring at Johnson Creek Commons.

Figure 7. Transect for bee monitoring at Yukon Bioswales.

Error! Bookmark not defined. defined.

Figure 8. BoxCox Power Transformation indicated that taking the reciprocal of data would result in a normalized distribution of the data. ......................................... 15

Figure 9. QQ Plot indicated that data was still not normalized after the reciprocal was taken.

Figure 10. Boxplot representing number of bee observations from entire season, separated out by site. Yukon Bioswales (YBIO), Johnson Creek Commons (JCC) and Beyer Court (BC). Bee observations were not found to be significant between sites.

Figure 11. Bee composition at Yukon Bioswales from entire monitoring season (June August). Bumblebee and Tiny dark bee were the two most predominant bee groups.

Figure 12. Bee composition at Johnson Creek Commons from entire monitoring season (June - August). Bumblebee, Tiny dark bee and striped sweat bee groups were the most predominant

Figure 13. Bee composition at Beyer Court from entire monitoring season (June August). Bumblebee, Tiny dark bee and Chap legged bee groups were the most predominant.

Figure 14. Percent cover floral resource composition at Yukon Bioswales for July. Data

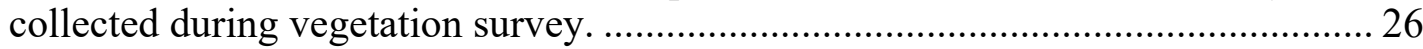

Figure 15. Percent cover floral resource composition at Yukon Bioswales for June. Data collected during vegetation survey. ................................................................... 26

Figure 16. Percent cover of floral resource composition at Yukon Bioswales for August.

Data collected during vegetation survey........................................................ 27

Figure 17. Percent cover floral resource composition at Beyer Court for June. Data

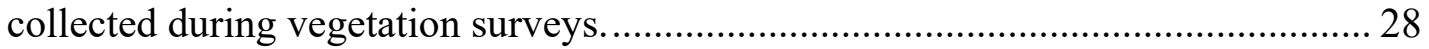

Figure 18. Percent cover floral resource composition at Beyer Court for July. Data collected during vegetation surveys................................................................... 29

Figure 19. Percent cover floral resource composition at Beyer Court for August. Data collected during vegetation surveys..........................Error! Bookmark not defined.

Figure 20. Percent cover floral resource composition at Johnson Creek Commons for July. Data collected during vegetation surveys.................................................. 30 
Figure 21. Percent cover floral resource composition at Johnson Creek Commons for July. Data collected during vegetation surveys.

Figure 22. Percent cover floral resource composition at Johnson Creek Commons for August. Data collected during vegetation surveys.

Figure 23. Average Native Bee Floral Resource Visitation from June-August on floral resources at all sites. Dark lines represent the mean number of native bees observed per observational period. The dashed lines represent the maximum number of native bees seen per observational period. The open circles represent outliers. Goldenrod was visited the most frequently, however typical "honey bee preferred plants" such as Milkweed had no native bee observations

Figure 24. Honey bee Floral Resource Visitation from June-August. Dark lines represent the mean number of honey bees observed per observational period. The dashed lines represent the maximum number of honey bees seen per observational period. The open circles represent outliers. Penstemons were visited the most frequently, however typical "honey bee pollinator plants" such as Milkweed had no observations.

Figure 25. Yukon Bioswale monitoring site in May prior to monitoring occurring. Site had full vegetation and numerous flowering plants.

Figure 26. Yukon Bioswale monitoring site in Mid July. Site was very dry and had greatly decreased floral diversity.

Figure 27. Yukon Bioswale monitoring site in August. Site was extremely dry and had very few flowering resources. 


\section{Introduction}

Bee pollinators can thrive in highly urbanized environments if their preferred floral resources and habitat types are available. Urbanized environments tend to be fragmented into small isolated patches of natural areas. These resulting urban fragments such as road edges, backyard gardens, and flower beds can provide habitat for bees in inhospitable anthropogenic environments (Westrich 1996). Threats to bees from urbanization, include loss of bare dirt for nesting habitat, loss of total green space, and loss of diverse floral resources available throughout the season. Currently, in cities such as Portland, Oregon there is a large push for sustainable urban planning including the creation of "pollinator friendly habitats". Establishing enhanced pollinator sites in cities may mitigate some threats bee pollinators are currently facing in urbanized environments, if the sites are properly planned and managed.

Creation of enhanced pollinator sites is not an easy task as there are many complex components in the creation of the entire system. Previous research has found that providing floral resources throughout the season with varied floral bloom times is extremely important for resource availability and bee seasonality (Wojcik et al., 2008). Enhanced pollinator sites are often constructed based off "pollinator plant lists" that aren't backed by scientific data and are usually not regionally specific lists. Additionally, pollinator plant lists are often created with only the honey bee in mind, although it has been indicated that native bees can provide a similar rate of pollination. Pollinator habitat in urbanized areas should focus on providing both habitat and floral resources for bee pollinators. Although there has been a substantial amount of research done on pollinator competition (Thomson 2004), there has been very little work on how small-scale local 
landscapes such as enhanced pollinator sites can affect pollinator visitation, specifically native bee visitation. The implications of pollinator decline on ecosystem functioning is crucial and research priorities for unraveling the multifactorial pressures on insect pollinators should include establishing a causal link between floral resource availability and pollinator abundance and diversity at landscape scales (Vanbergen et al., 2013).

Native bees are extremely important to our ecosystem, simulation results have predicted that native bees provided sufficient pollination at $>90 \%$ of farms studied, when compared to honeybee pollination (Winfree et al., 2007). Native bees provide an important and free alternative to honey bees. Providing floral resources and habitat for native bee pollinators in the city is crucial with the recent decline of honey bees associated with colony collapse disorder (CCD), ridovirus and microsporidian (Bromenshenk et al., 2010).

Native bee populations can be supported in urban areas if appropriate floral and habitat resources are provided to them. Floral resources for pollinators in particular can be poorly provided for due to urbanization and resulting habitat fragmentation in cities. Floral resources are necessary for pollinators nutritional demands (Cannon \& Horton 2009). Honey bee floral resource preferences have been studied quite extensively due to their use in the agricultural sector for pollination (Cook et al., 2003 ; Ramalho et al., 1990 ; Hawkins et al., 2015). Interactions between honey bees and native bees on floral resources has also been studied (De Menezes Pedro \& De Camargo 1991). However, native bee floral resource preference has not received the attention needed, with only one main study focusing on native bee preferences (Jha \& Kremen 2013).

There are a few floral resources that are often associated with being "pollinator plants", such as Asclepias speciosa, or Ceanothus species. However, no research 
specifically identifying milkweed or wild lilac as preferred floral resources for native bees has emerged. A study done in Vancouver, British Columbia, Canada indicated that in highly urbanized environments, weeds such as Taraxacum species had the highest diversity of bee visitors (Tommasi et al., 2004). The idea that native bees prefer weedy species is not novel, but it has recently been found that weed abundance also improved the survival of other wild flora and increased socio-cultural value of the landscape (Bretagnolle \& Gaba, 2015). Interestingly, it is thought that honey bee pollination contributed very little to the success of most weedy species (Huryn 1997) indicating that native bees may play a specialized role in weed pollination.

Native or invasive status of floral resources plays a role in bee visitation when determining what floral resources to plant at enhanced pollinator sites. Tepedino et al., (2008) found that on average, in Utah, invasive floral resources were visited by twice as many bee species compared to native floral resources. However, visitation and native or invasive status appeared to be landscape specific as Nienhuis et al., (2009) found no impact of invasive plants on standardized pollinator abundance in Ireland. Due to the differences found in research about the significance of native or invasive floral resource status and bee visitation, variation in findings may indicate that bee's floral resource preferences are determined by local pressures and the local landscape.

In addition to floral resource choice, it has been found that bees foraging responses depends on spatial scale and that habitat composition had a stronger effect than distance to the nearest forest fragment on bee presence (Jha et al., 2009). In addition to small-scale floral resources, the importance of dense floral resources to bees has been shown previously as bees appeared to act as optimal foragers on a small community scale because they were more heavily influenced by the frequency of floral resources (floral 
density) rather than the suite of different species of floral resources (floral diversity) (Hegland \& Boeke 2006). The large majority of bees in the United States nest in the ground and need bare uncompacted dirt for nesting habitat, which is rare to find in the city (Cane 1991). However, some small urban isolated patches such as designated pollinator habitat can increase the available ground nesting habitat in the area and create refuge in the city for ground nesting bees (Hernandez et al. 2009). In addition to providing for ground nesting bees, urban fragments converted to pollinator habitat can increase habitat connectivity which is beneficial for most terrestrial species (Beninde et al., 2015).

Portland, Oregon is a highly urbanized environment which has also been noted to be a very environmentally friendly city (Podobnik 2011). Many environmentally aware residents of Portland have created "pesticide free zones" and "bee friendly gardens" within their own yards and neighborhoods. Creating "green" or "pollinator friendly" areas in the city is challenging, due to the lack of scientifically sound "local pollinator plant lists", lack of knowledge about bee species in the local area as well as lack of knowledge about appropriate local habitat for bees. Luckily, Portland residents who are practicing these activities on their own are joined in the effort by non-profit organizations such as Green Lents, as well as passionate scientists who are looking to answer these questions. The aim of this project was to address the challenges of implementing pollinator habitat by monitoring bees in order to provide regionally specific planting recommendations for native and honey bees in South East Portland, provide bee monitoring data as well as host several community science workshops to increase knowledge about bees and their habitats. 
Due to the importance of available late season floral resources for bees, I specifically was interested if diverse floral resources were being offered throughout the monitoring season (including late season plants) at enhanced pollinator sites which were planted with different "pollinator plant lists". There has been previous research indicating little to no competition for food resources occurs between honey bees and native bees (Thomson 2004), I was interested if there was a dietary overlap between honey bees and native bees. The aim for my thesis project was determining if we were providing the most preferred floral resources at enhanced pollinator sites for bees, if floral resources were available throughout the season, and if dietary preferences between native and honey bees would allow for the identification of "native bee floral resources" in South East Portland.

\section{Hypotheses}

1. What predicts bee presence at enhanced pollinator sites?

H1: Blossom density will be a significant predictor of bee presence because floral resources with higher blossom densities can provide more resources to bees (Hegland \& Boeke 2006).

2. At enhanced sites, are diverse floral resources being offered throughout the season to support urban bee seasonality?

$\mathrm{H} 2$ : There will be a difference in the number of bee observations between June, July and August due to differences in availability of diverse floral resources at sites.

3) Is there a dietary preference overlap between native and honey bees?

H3: There will be difference between dietary preference of honey bees and native bees. 


\section{Methods}

Site selection

Green Lents a nonprofit organization in Lents, Oregon started a "Pollinator Habitat Program" initiative that focused on increasing pollinator friendly habitat in conjunction with bioswales and raingardens in Lents, Oregon in 2012. Part of this initiative was to convert $3,500 \mathrm{sq} f \mathrm{ft}$ of weedy and paved area to pesticide-free pollinator habitat area, Green Lents is currently working on expanding designated pollinator habitat to multiple areas in South East Portland. Green Lents had a history of being exceptionally involved in community activities in the Lents neighborhood, which I viewed as an opportunity to incorporate community members, and thereby community science into my thesis project.

The inclusion of community science members in this project was very important to me. I believed that pollinator education and outreach needed to be achieved everywhere, especially in historically underserved neighborhoods such as Lents. Pollinator research is a fantastic area to involve community science members in, as the amount of monitoring data can be quadrupled with the utilization of community members, compared to a single scientist alone. Two out of the three enhanced sites were located outside ROSE Community Development housing, which offered reduced pricing and mixed-use apartment communities. I had anticipated that choosing sites near ROSE housing would be advantageous for recruiting volunteers.

At the time of this thesis project, Green Lents had three enhanced sites that were established enough to have monitoring conducted on them; Beyer Court Rain Garden, SE Yukon Bioswales and Johnson Creek Commons Rain Garden. Johnson Creek Commons had a total area $186.81 \mathrm{ft}(56.94 \mathrm{~m})$, Beyer Court Rain Gardens had a total area of 
$1,026.51 \mathrm{ft}^{2}\left(95.37 \mathrm{~m}^{2}\right)$ and Yukon Bioswales total area was $952.02 \mathrm{ft}^{2}\left(88.4 \mathrm{~m}^{2}\right)$, each individual bioswale consisted of an area of $317.34 \mathrm{ft}(96.7 \mathrm{~m})$ (Figure 1). Each site was dominated by varied vegetation as Green Lents used different "pollinator plant lists" (Appendix A) to establish plants on the three sites. These enhanced pollinator areas also provided ecosystem services by functioning as storm water retention areas and rain gardens. Yukon Bioswale sites mostly consisted of smaller plants such as Silene coronaria and Penstemon species (Figure 2), Beyer Court site had a mixture of small and medium sized floral resources such as Eschscholzia californica and Cornus sericea (Figure 3), and the Johnson Creek Commons site had larger floral resources than the other sites such as Mahonia aquifolium and Lonicera involucrata (Figure 4).

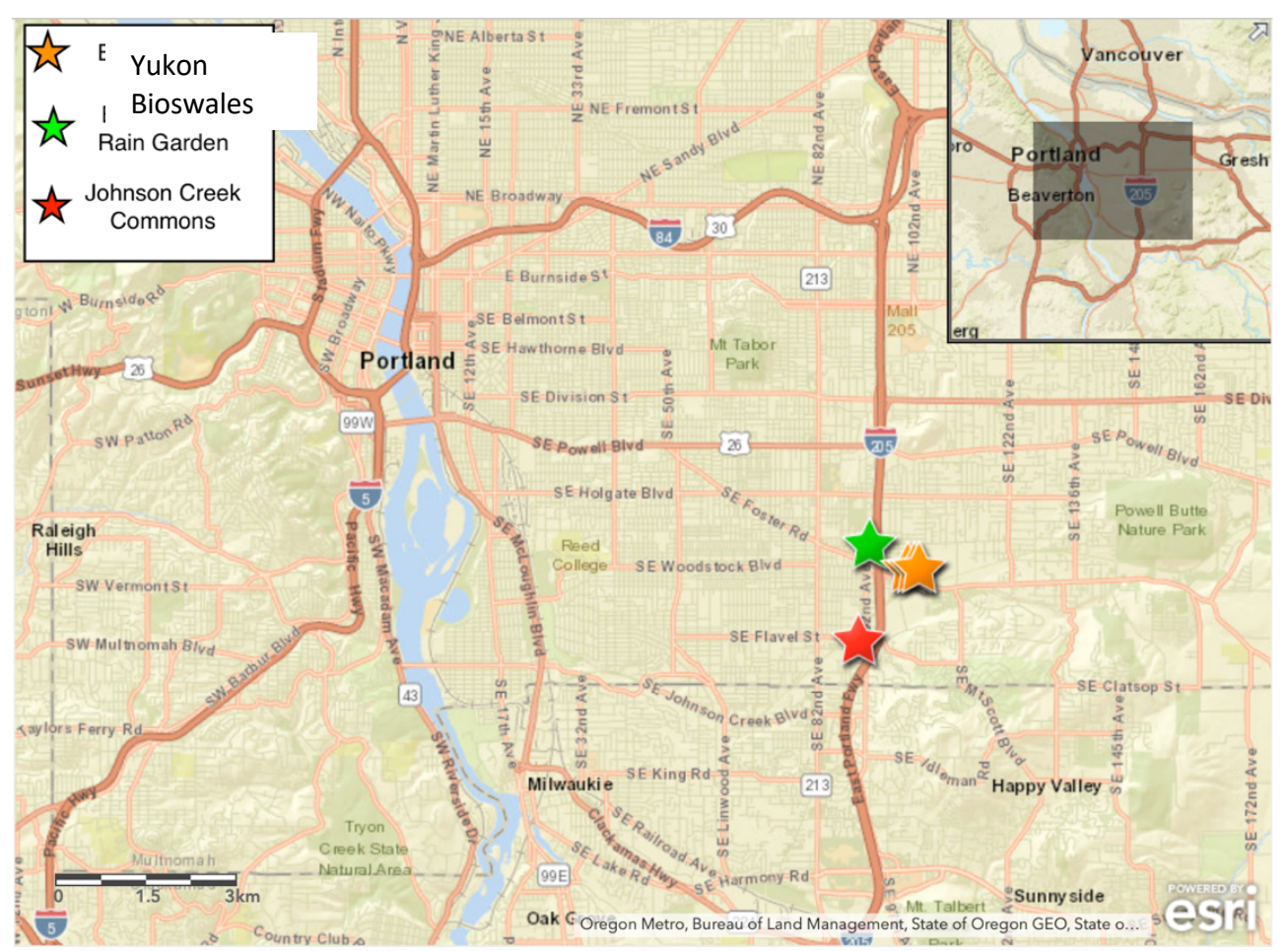

Figure 1. Map of monitoring locations in Lents, Oregon. Yukon Bioswales (3) are indicated by orange stars. Beyer Court Rain Garden indicated by the green star. Location of Johnson Creek Commons indicated by the red star. 


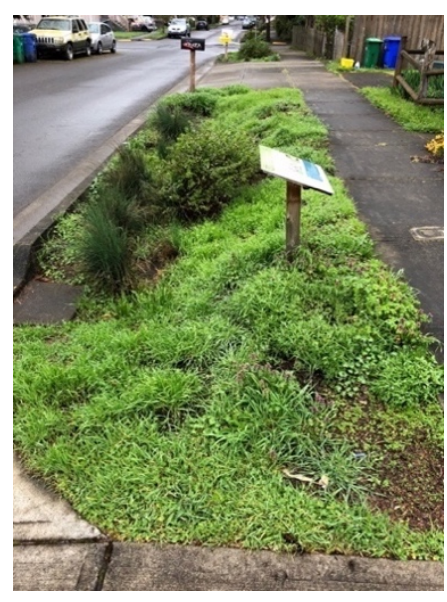

Figure 2. Yukon Bioswales monitoring site in Lents, OR. Photo taken in May prior to monitoring period.

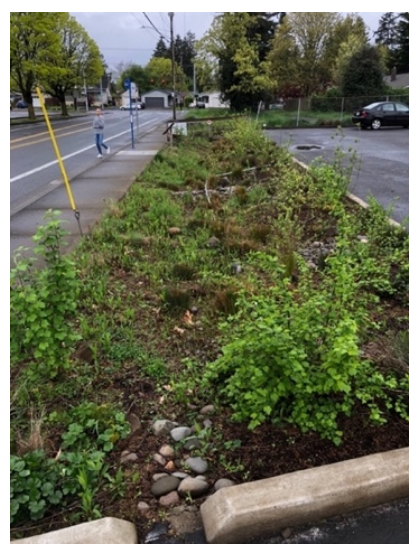

Figure 3. Beyer Court Rain Garden monitoring site in Lents, OR. Photo taken in May prior to monitoring period.

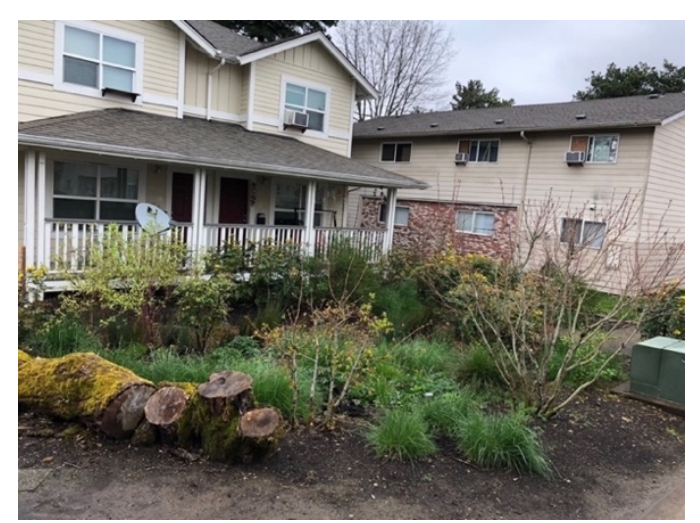

Figure 4. Johnson Creek Commons monitoring site in Lents, OR. Photo taken in May prior to monitoring period. 


\section{Community scientists}

Community science members were utilized in gathering observational bee data and recruited from January to May 2018 by way of "Bee Count PDX" Facebook posts detailing training session information which were shared by multiple entities, such as the East Multnomah Soil and Water Conservation District (EMSWCD), Green Lents and Portland Urban Beekeepers. Additionally, flyers were handed out while attending multiple EMSWCD workshops about "beneficial insects" and "planting for pollinators" as well as at multiple locations around Portland State University.

From those interested in signing up how to identify bees, an application was completed which assessed basic bee knowledge, which site they lived closest to, if they had prior monitoring experience with the community science protocol and if they would be willing to commit to monitoring pollinators twice a month from June to August, All participants who filled out an application were invited to participate in BeeCountPDX regardless of experience.

Two training sessions were held in order to teach community scientists about the importance of community science, and how to identify and monitor bees at the enhanced sites as well as how to identify and record floral resources for each bee observation. Each training session lasted about three hours, and included assessing scientific literacy, general bee biology and knowledge of Portland's urban bees. Additionally, community members were taught how to identify bees into twelve morphogroups using the Maritime Citizen Science Monitoring guide (Minnerath et al., 2014), as well as how and what to record when at monitoring sites. Due to funds received from the Edward D. and Olive C. Bushby scholarship, community members were provided with a bee vacuum which was 
theirs to keep at the end of the season, full color bee monitoring guides, full color floral resource identification tables made by myself as well as pre-printed observational logs (Appendix B, C). Additionally, in order to maintain a sense of friendship and comradery drinks and snacks, were provided to the volunteers during the training sessions.

\section{Monitoring protocol \& data collection}

The Xerces' Maritime monitoring guide for citizen scientists was used (Minnerath et al., 2014). This monitoring protocol split bees into ten "morphogroupings" which combined bees with similar morphological characteristics, not by species or genera. Bees are grouped by physical characteristics such as body shape, color, location of pollen collecting hairs, and antennae size. I used the Xerces' guide because I wanted to involve community scientists and thought that it was the "easiest" guide to follow to learn how to identify native bees for nonprofessional community members. Additionally, a monitoring guide needed to be selected that would be quick for community members to learn and not involve too much extra information that would overwhelm people and cause them not to be involved in the project in their free time.

All three sites were monitored by volunteers at least once a month. A google calendar was created so that volunteers could upload their monitoring schedule, which ensured that sites were not getting monitored repeatedly on the same day by different volunteers. I monitored each site weekly, on a random day at random times, between 10$3 \mathrm{pm}$.

Using data collection protocol outlined by the Xerces guide, volunteers were to monitor if the temperature was above $60^{\circ} \mathrm{F}$, winds less than six mph (light breeze) and enough sunlight to see your shadow, typically 10 am to 3 pm (Minnerath et al., 2014). 
Community scientists were told to walk transects through sites at a pace of 10 feet per minute, only recording data on bees visiting the reproductive parts of the flower. This equated to about 15 minutes per site, walking both sides of the transect line at Beyer Court (Figure 5) and Yukon Bioswales (Figure 7) as well as monitoring the inner and outer radius of Johnson Creek Commons (Figure 6). Volunteers could either use a bug vacuum, nets, or visual observation to determine which one out of the ten groups the bee belonged to and its associated floral resource at the time of observation. Community scientists were encouraged to record other pollinator observations such as bee flies or wasps, although this data was not included in analysis due to low numbers of recorded observations.

Data was then entered into Google sheets, where the volunteers had access to see the entire dataset. All volunteers "turned in" data sheets at the end of the collection season and had the opportunity to be able to enter in their own monitoring data and look at the data collected as we moved throughout the project. Two individuals entered in their own data, and three other volunteers gave me their observational sheets to enter in. Volunteers were contacted throughout the monitoring season (June - August) with updates about the project, as well as setting up guided monitoring sessions. Updates were also posted to BeeCountPDX Facebook page to increase interest and participation throughout the thesis writing portion and defense of this thesis project. 


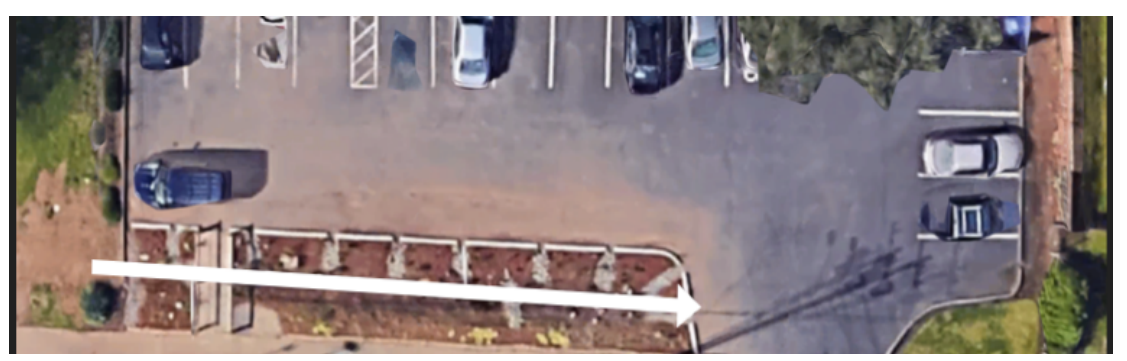

Figure 5. Transect for monitoring at Beyer Court Rain Garden.

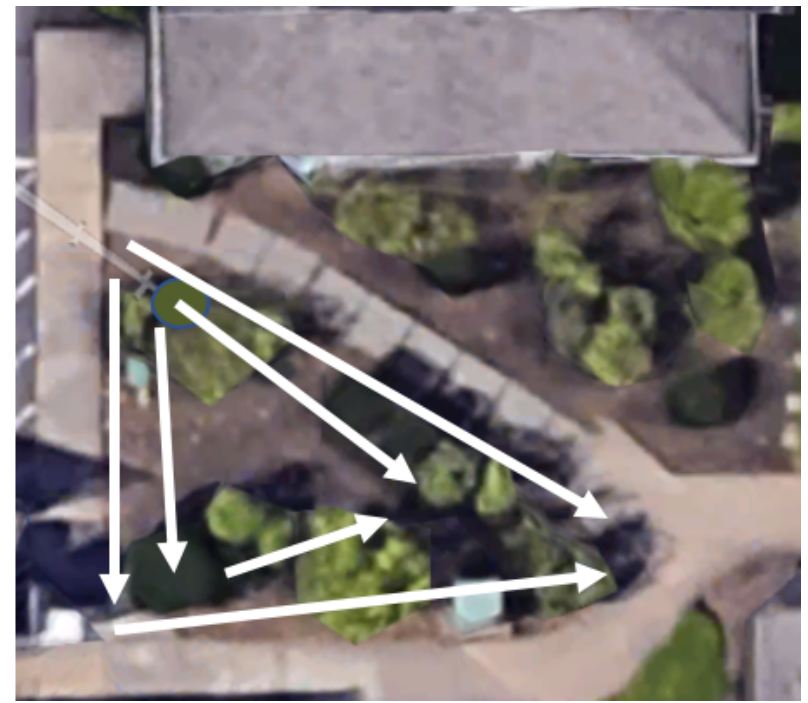

Figure 6. Transects (outer and inner) for bee monitoring at Johnson Creek Commons.

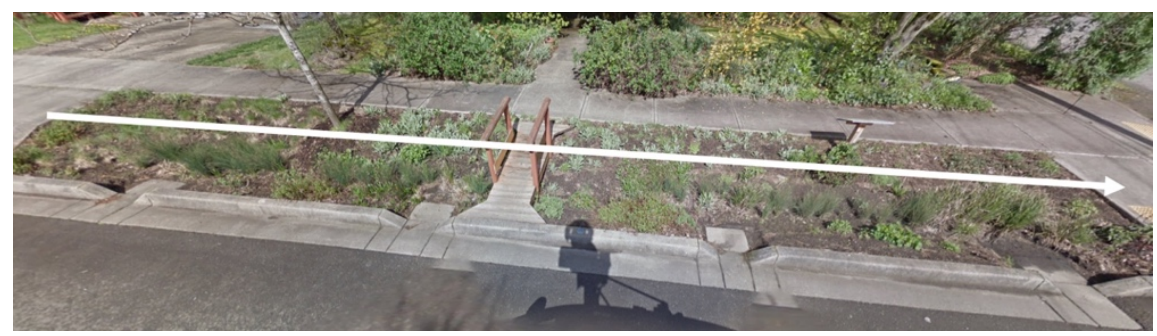

Figure 7. Transect for bee monitoring at Yukon Bioswales. 


\section{Vegetation surveys}

Vegetation surveys were completed once a month at each site, with correlated monitoring in order to be able to assess the relationship between small scale vegetation metrics and pollinator visitation. Vegetation surveys were completed by me around the second week of the month at each site $(6 / 13,7 / 17,8 / 22)$ in order to correctly incorporate data for all flowering plants for the month, per site. At each site, for each flowering plant species each month; density, cover and frequency were calculated. In order to determine the proportional representation of each species relative to the entire flowering plant community, relative cover, relative density and relative frequency values were computed (Baxter 2014). Relative cover was calculated per species by taking the flowering species cover (total area of the entire plant) and dividing by total percent cover at the site. Relative density was calculated by taking the number of individuals per species and diving it by the total number of individuals at sites. Relative frequency was calculated by taking the number of quadrats that the species occupied and dividing it by the total number of quadrats at sites. The methods used (Hegland \& Boeke 2006) to measure individual inflorescence blossom density involved measuring inflorescence size, depending on blossom type. If the blossom was circular, the radius of the inflorescence was measured, and if the blossom was tubular the length and width of the inflorescence was measured.

I measured inflorescence blossom density on twenty individuals of the same flowering species at each site, each month in order to calculate average inflorescence blossom density per species per month. Inflorescence blossom density was calculated by taking the average number of inflorescences per species and multiplying it by the averaged inflorescence area. Vegetation surveys were completed on the entire monitoring 
area in order to correlate monitoring data with vegetation data. Only floral resources that were flowering during vegetation surveys were recorded and used for data analysis.

\section{Statistical Analysis Methods}

\section{Data structure}

Because each site was visited by volunteers in addition to myself, only bee monitoring data that I collected within a two-week period were used to coordinate plant vegetation metrics and bee presence. Data were grouped this way in order to avoid false generalizations about floral vegetation metrics such as blossom density which changed throughout the month and the season (Chmielewski et al., 2004). Whole season data set was used to assess floral resource available and site availability as well as to find differences between preferences of floral resources at sites. All data was analyzed using R software (R Core Team) using the packages Lme4 (Bates et al., 2015), MASS (Venables \& Ripley 2002), Dyplr (Wickham et al., 2018) and Lattice (Deepayan 2008). Normality

Due to the nature of the data being observational, the data did not follow a normal distribution. A Boxcox Power Transformation test was used in order to determine what value to transform lambda with in order to achieve normality (Osborne 2010). The boxcox test indicated that transforming the data by taking the reciprocal would result in normality (Figure 8). The reciprocal of the data was taken, and the data still did not follow a normal distribution (Figure 9). Data were then logged, and square rooted however, these additional statistical transformations did not result in the data following a normal distribution. Due to the failure to normalize data with simple transformations, I decided to pursue non parametric statistical tests in order to avoid transforming the 
variable and having additional variance on the transformed variable. Because of the non-normalized data, I used a generalized linear mixed model (GLMM) to evaluate my data. A GLMM is the non-parametric version of a logistic regression. GLMM's are complex and can be often misused in ecological work, I relied heavily on a GLMM model fitting tree created by Bolker et al., (2009) or model parameters and model selection.

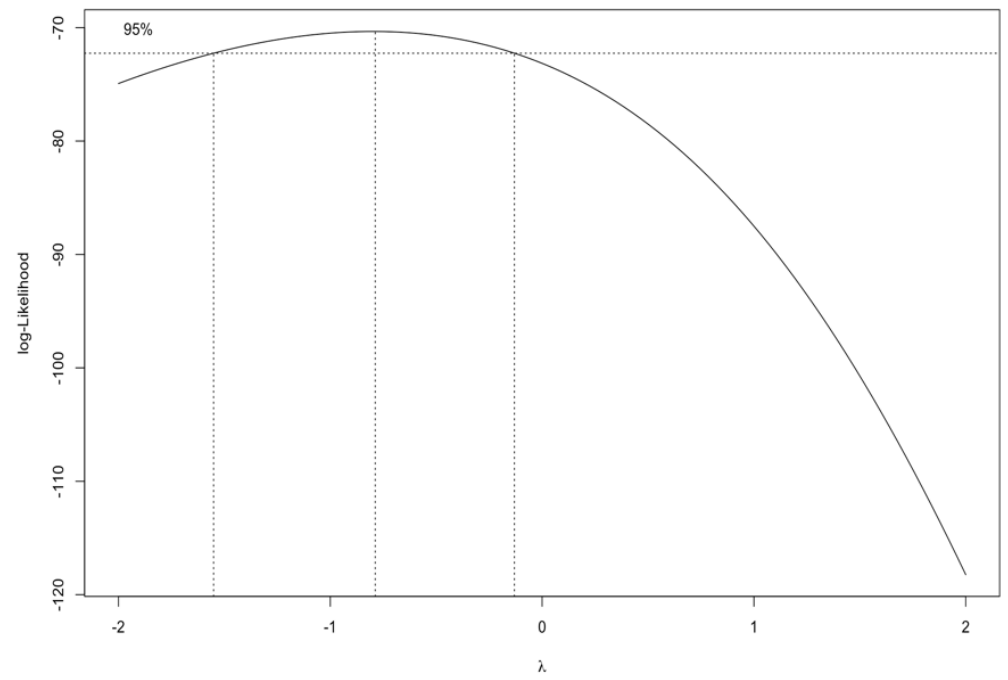

Figure 8. BoxCox Power Transformation indicated that taking the reciprocal of data would result in a normalized distribution of the data. 


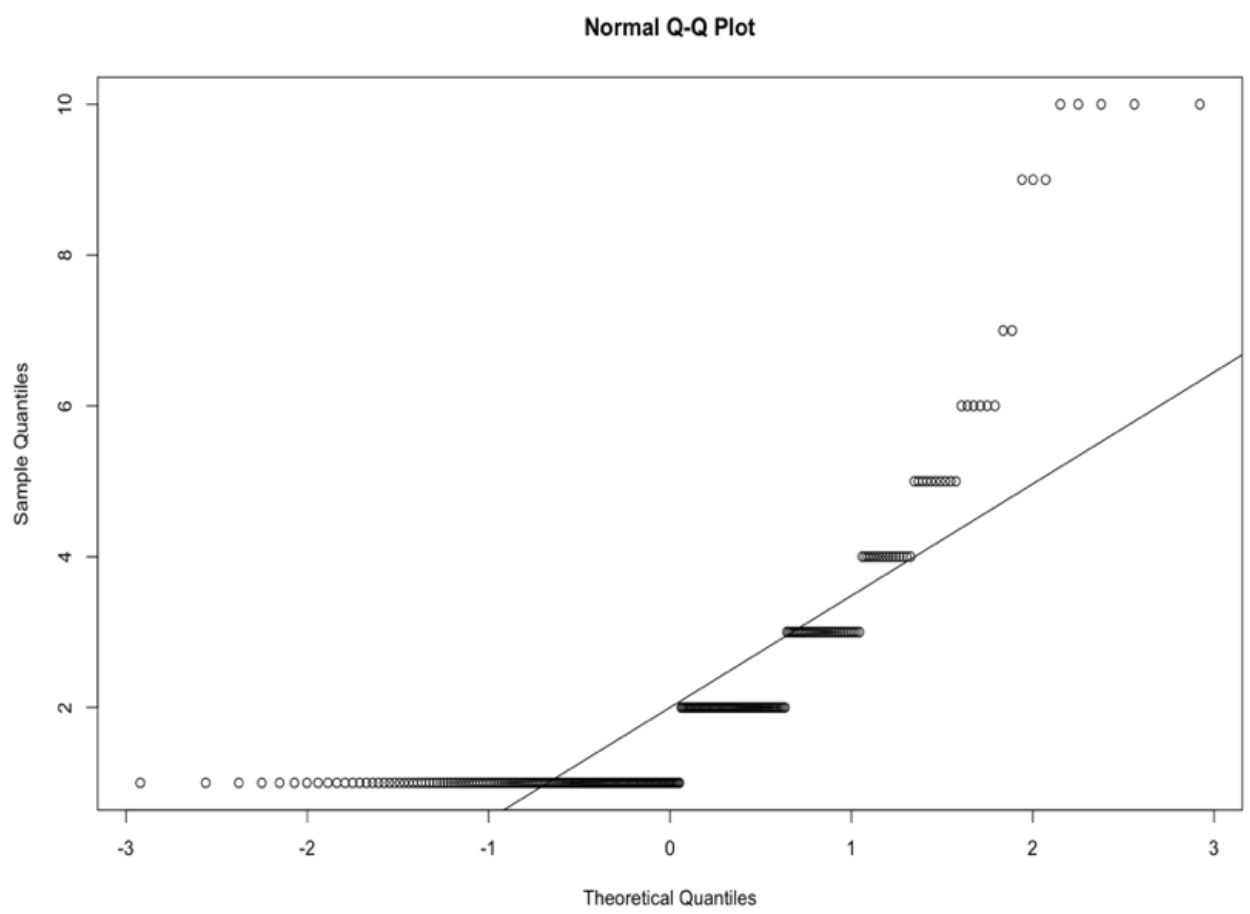

Figure 9. QQ Plot indicated that data was still not normalized after the reciprocal was taken.

\section{Predictor variables for GLMM}

Predictor variables for bee presence were divided into three main groups; floral resources, floral resource metrics, and morphospecies group of bee (Table 1). Floral resources included all flowering plants that were at sites recorded by vegetation surveys. Floral resource metrics were measured and calculated once a month, per site and morphospecies groups of bees are those identified by the monitoring protocol (Minnerath et al., 2014). Non numeric data were converted into factors with different levels in order to compare numeric and character data. 
Table 1. Predictor variables used for predicting bee presence at sites using a GLMM model. Variables were broken into three main sections, floral resources at all sites, floral resource metrics that were measured once a month per site, and morphospecies bee group.

Floral Resources

Blanket flower
Bluebell
California poppy
Checkermallow spp.
Dogwood
Douglas aster
Douglas spirea
Fireweed
Goldenrod
Milkweed
Nodding onion
Nootka rose
Oregon grape
Oregon sunshine
Penstemon spp.
Rose campion
St. John's wort
Twinberry honeysuckle
White clover
Wild lilac

\section{Bee Morphospecies Groups}

Bumblebee

Chap legged bee

Cuckoo bee

Honey bee

Medium dark bee

Metallic green bee

Striped hairy belly bee

Striped sweat bee

Tiny dark bee

\section{Floral Resource Metrics}

Blossom density

Blossom type

Cover

Density

Frequency 


\section{Coding predictor variables}

Non numeric data were converted into factors with different levels in order to compare numeric and character data. Vegetation metrics that were converted into factors included: site, bee, type, floral resource, month, blossom type, and status (invasive or native vegetation). $\mathrm{R}$ converted characters into numeric codes that corresponded to different factor levels. For example, bee group observed were converted into numeric values with different factor levels, meaning that instead of using "Bumblebee", the statistical program coded all "Bumblebees" into "1", "Striped Sweat Bees" into "2", etc. The conversion of character values allowed for the comparison between numeric and now factored data for model validation.

\section{Choosing a distribution}

I used penalized quasilikelihood (PQL) rules of thumb to define distributions for GLMM inference (Breslow 2004) in order to determine what distribution would best fit my data. Because the calculated mean of the independent variable (bee observations) was less than 5 (1.83) a Poisson distribution was used for fitting and interference of the GLMM for my data (Bolker et al., 2009). Sites were identified as the random effect. Due to the data having a low numeric mean of the independent variable, and only one random effect, Gaussian Hermite Quadrature (GHQ) approximation could be used to fit the data. The final model used for data analysis was a generalized linear mixed model (GLMM) fit by maximum likelihood (Adaptive Gauss-Hermite Quadrature, with 100 iterations of the model.

\section{Hypothesis testing statistic}

Overdispersion of the model was calculated by number of variance parameters, residual degrees of freedom, and Pearson residuals of the model (Harrison 2014). Testing 
the model for overdispersion resulted in a $p$-value of 0.9 this $(p$-value $>0.05)$ confirmed the data were not overdispersed within the model. Because the model was not overdispersed and did not need to account for a high level of uncertainty Wald Z, and X2 tests were used for hypothesis testing and identifying significant predictors of bee presence. Cooks D for influential data points (Pinho et al., 2015) was calculated and no significant outliers were identified. The final parameter used for GLMM fitting and inference was AIC (Burnham \& Anderson 2004). The AIC of the full model was 716 and AIC of the reduced model was 714, indicating a better fit with the reduced model.

\section{Testing other significant relationships}

Kruskal Wallis tests are non-parametric versions of one-way ANOVA's and are used when the assumption of normality of an ANOVA cannot be met. Kruskal Wallis test were implemented because data did not follow a normal distribution, and observations were independent (Vargha \& Delaney 1998). 


\section{Results}

\section{Urban Bee Composition}

A total of 566 individual bees were observed, with random monitoring occurring from June to August. The number of bee observations for the entire data collection season were similar between sites, Beyer Court had 185 bees observed, Johnson Creek Commons had 182 and Yukon Bioswale sites had 199 individual bees observed during the data collection season (Table 2). On average, more bees were seen per monitoring session at Johnson Creek Commons than Beyer Court or Yukon Bioswale sites but were not statistically different from each other. (Figure 10).

Table 2. Table displays numbers of bee observations of different bee groups from the entire data collection season (June, July, August). Johnson Creek Commons (JCC), Beyer Court (BC) and Yukon Bioswales (YBIO).

\begin{tabular}{|c|c|c|c|c|c|}
\hline \multicolumn{2}{|c|}{ JCC } & \multicolumn{2}{|c|}{ BC } & \multicolumn{2}{|l|}{ YBIO } \\
\hline Bumblebee & 36 & Bumblebee & 42 & Bumblebee & 78 \\
\hline $\begin{array}{c}\text { Chap legged } \\
\text { bee }\end{array}$ & 19 & $\begin{array}{c}\text { Chap legged } \\
\text { bee }\end{array}$ & 29 & Chap legged bee & 4 \\
\hline Cuckoo bee & 0 & Cuckoo bee & 3 & Cuckoo bee & 1 \\
\hline Honey bee & 16 & Honey bee & 25 & Honey bee & 11 \\
\hline $\begin{array}{l}\text { Medium dark } \\
\text { bee }\end{array}$ & 6 & $\begin{array}{l}\text { Medium dark } \\
\text { bee }\end{array}$ & 6 & $\begin{array}{l}\text { Medium dark } \\
\text { bee }\end{array}$ & 0 \\
\hline $\begin{array}{l}\text { Metallic green } \\
\text { bee }\end{array}$ & 4 & $\begin{array}{l}\text { Metallic green } \\
\text { bee }\end{array}$ & 8 & $\begin{array}{c}\text { Metallic green } \\
\text { bee }\end{array}$ & 13 \\
\hline $\begin{array}{l}\text { Striped hairy } \\
\text { belly bee }\end{array}$ & 1 & $\begin{array}{l}\text { Striped hairy } \\
\text { belly bee }\end{array}$ & 10 & $\begin{array}{l}\text { Striped hairy } \\
\text { belly bee }\end{array}$ & 8 \\
\hline $\begin{array}{c}\text { Striped sweat } \\
\text { bee }\end{array}$ & 32 & $\begin{array}{c}\text { Striped sweat } \\
\text { bee }\end{array}$ & 17 & $\begin{array}{c}\text { Striped sweat } \\
\text { bee }\end{array}$ & 17 \\
\hline Tiny dark bee & 68 & Tiny dark bee & 45 & Tiny dark bee & 67 \\
\hline Total & 182 & Total & 185 & Total & 199 \\
\hline
\end{tabular}




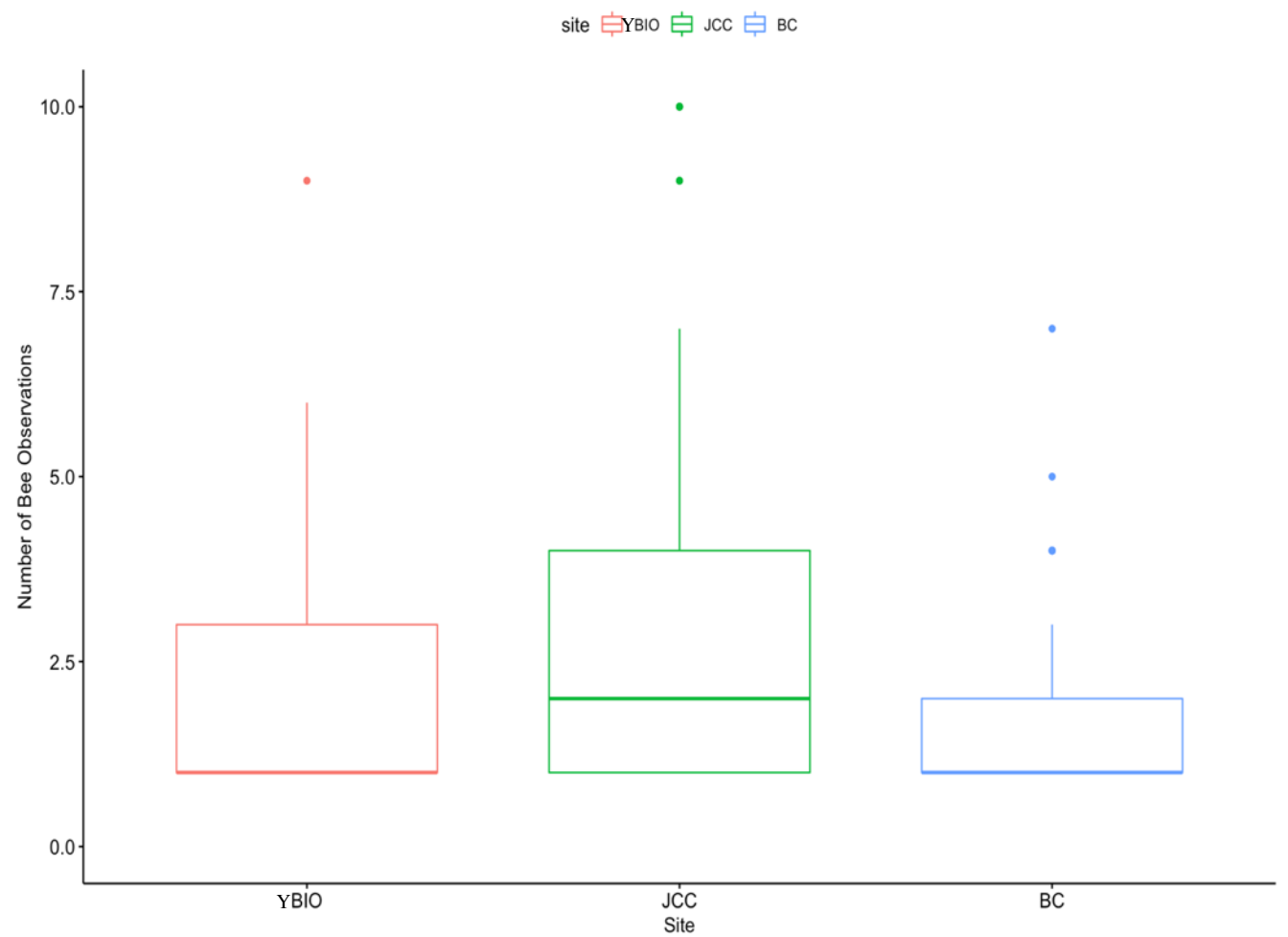

Figure 10. Boxplot representing number of bee observations from entire season, separated out by site. Yukon Bioswales (YBIO), Johnson Creek Commons (JCC) and Beyer Court (BC). Bee observations were not found to be significant between sites.

Overall, the most abundant bees were similar at all sites, with tiny dark bees and bumblebees making up the large majority of the urban bee composition at all three sites. However, other bee groups varied between sites. No medium dark bees were observed at the Yukon Bioswales, and no cuckoo bees were observed at Johnson Creek Commons. Honey bee composition varied between sites but, was very low compared to native bee groups fluctuating between $5-16 \%$ of bees monitored at sites (Fig. 11,12,13). 


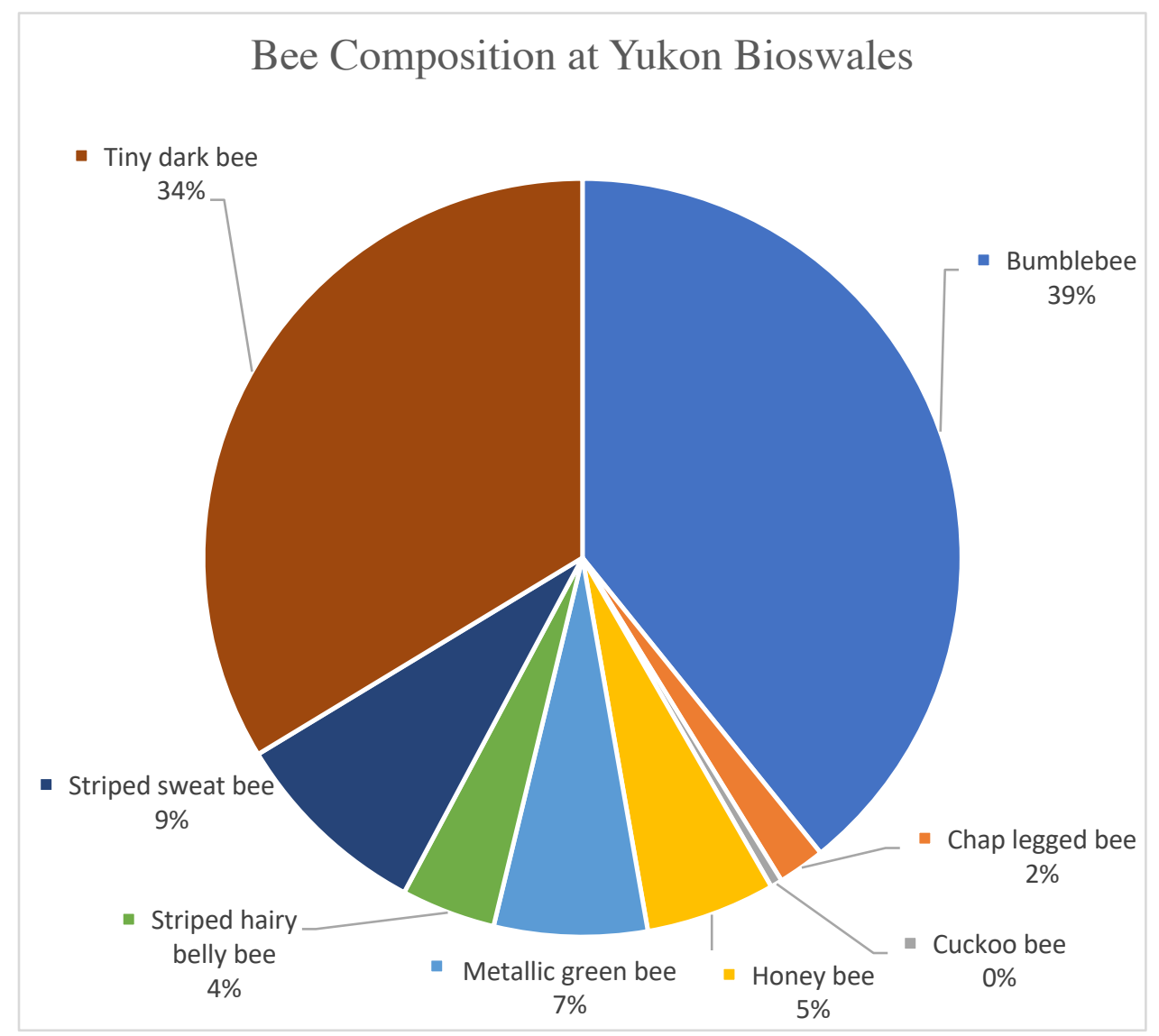

Figure 11. Bee composition at Yukon Bioswales from entire monitoring season (June - August). Bumblebee and Tiny dark bee were the two most predominant bee groups. 


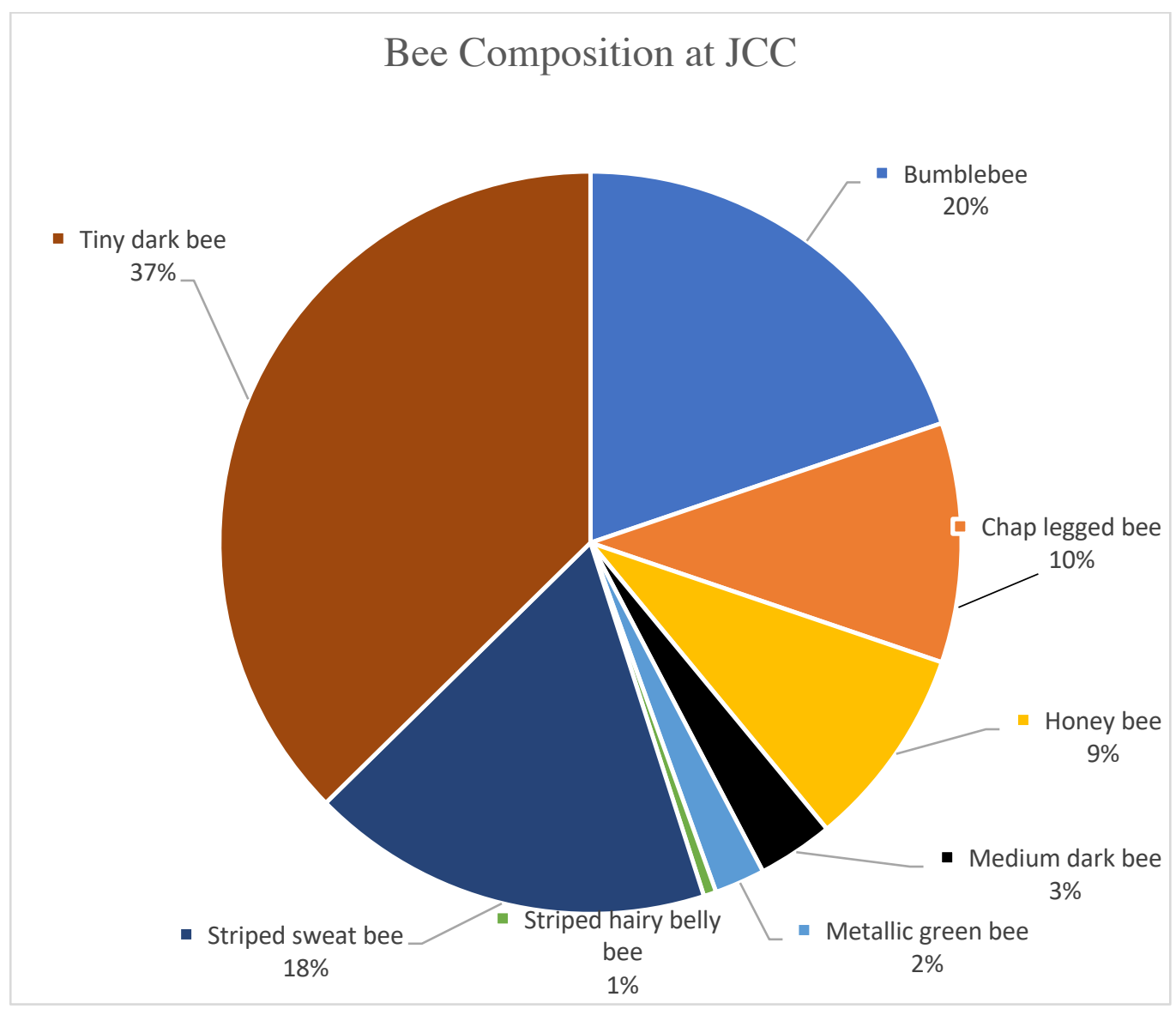

Figure 12. Bee composition at Johnson Creek Commons from entire monitoring season (June - August). Bumblebee, Tiny dark bee and. striped sweat bee groups were the most predominant. 


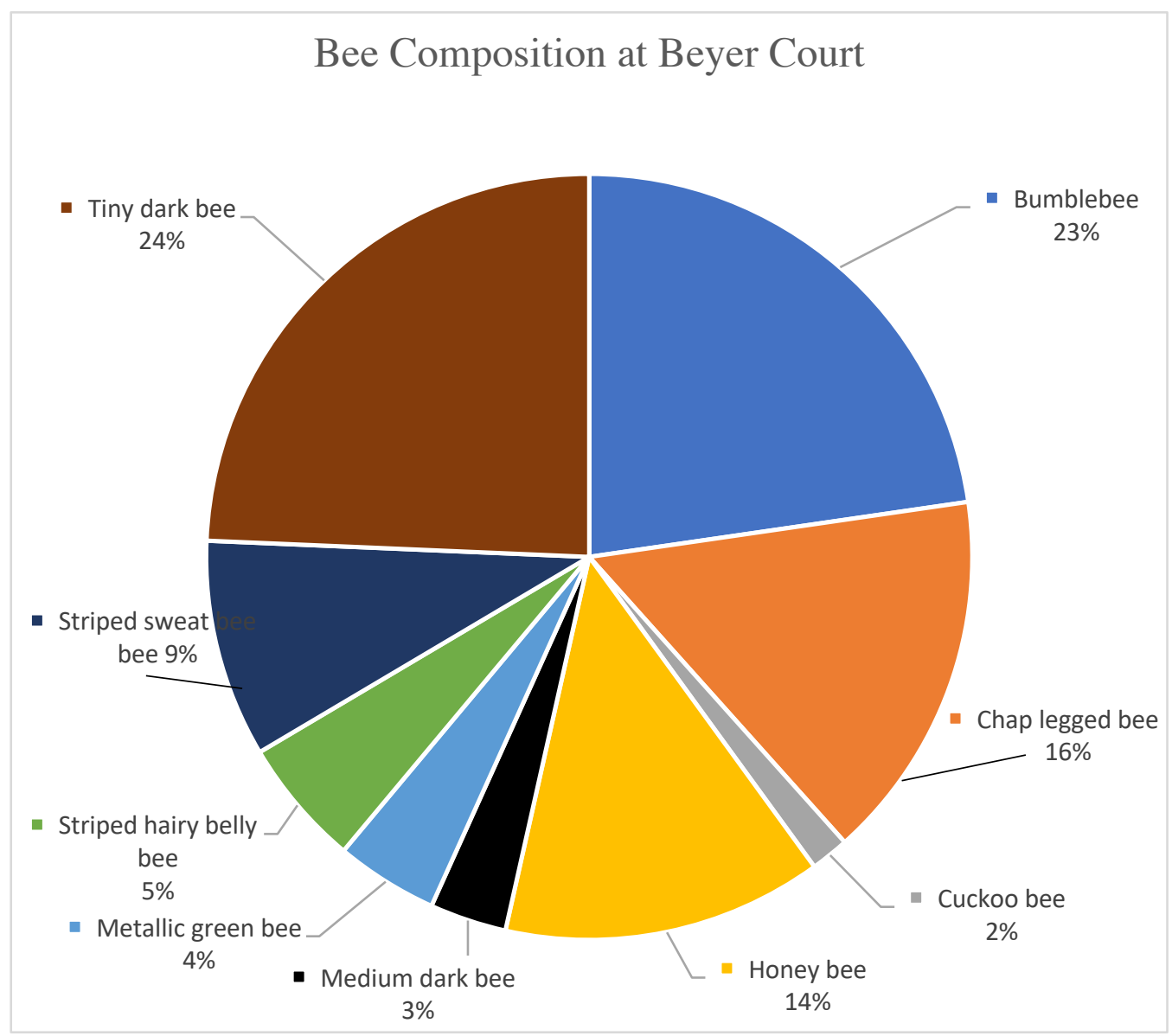

Figure 13. Bee composition at Beyer Court from entire monitoring season (June - August). Bumblebee, Tiny dark bee and Chap legged bee groups were the most predominant. 


\section{Floral Resource Composition}

Floral resource composition varied between sites as well as between months.

Floral species richness also varied, sites had high species richness during June and greatly decreased species richness in August (Table 3).

Table 3. Floral species richness measured by number of species flowering during vegetation surveys at Beyer Court (BC), Johnson Creek Commons (JCC) and Yukon Bioswales (YBIO) for the monitoring season June, July and August.

\begin{tabular}{|c|c|c|c|}
\hline & June & July & August \\
\hline BC & 16 & 6 & 3 \\
\hline JCC & 12 & 6 & 4 \\
\hline YBIO & 8 & 9 & 6 \\
\hline
\end{tabular}

\section{Yukon Bioswales}

Overall the Yukon bioswale sites had less floral resource species richness compared to Beyer Court and Johnson Creek Commons sites (Table 3). In June, nodding onion and California poppy were the predominant available resources for bees (Figure 14). In July, the species richness of the Yukon Bioswales increased by one species (Table 2). The floral resource composition shifted from being mostly dominated by California poppy (Figure 14) to penstemon and nodding onion being the predominant resources (Figure 15). In August, floral resource species richness decreased at Yukon bioswales (Table 3). Floral composition shifted to being dominated by Douglas aster and penstemon species (Figure 16). 


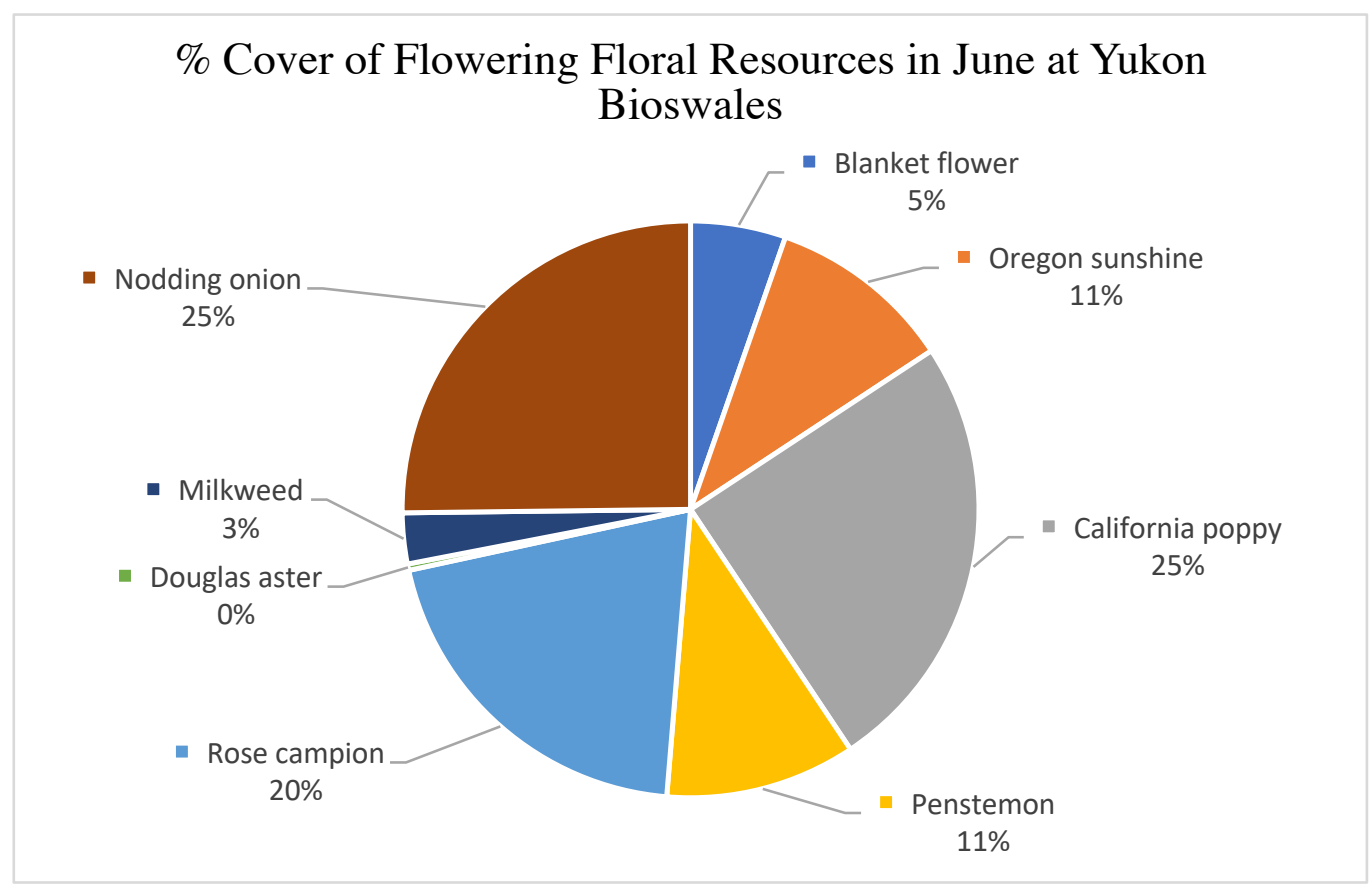

Figure 14. Percent cover floral resource composition at Yukon Bioswales for July. Data collected during vegetation survey.

\% Cover of Flowering Floral Resources in June at Yukon

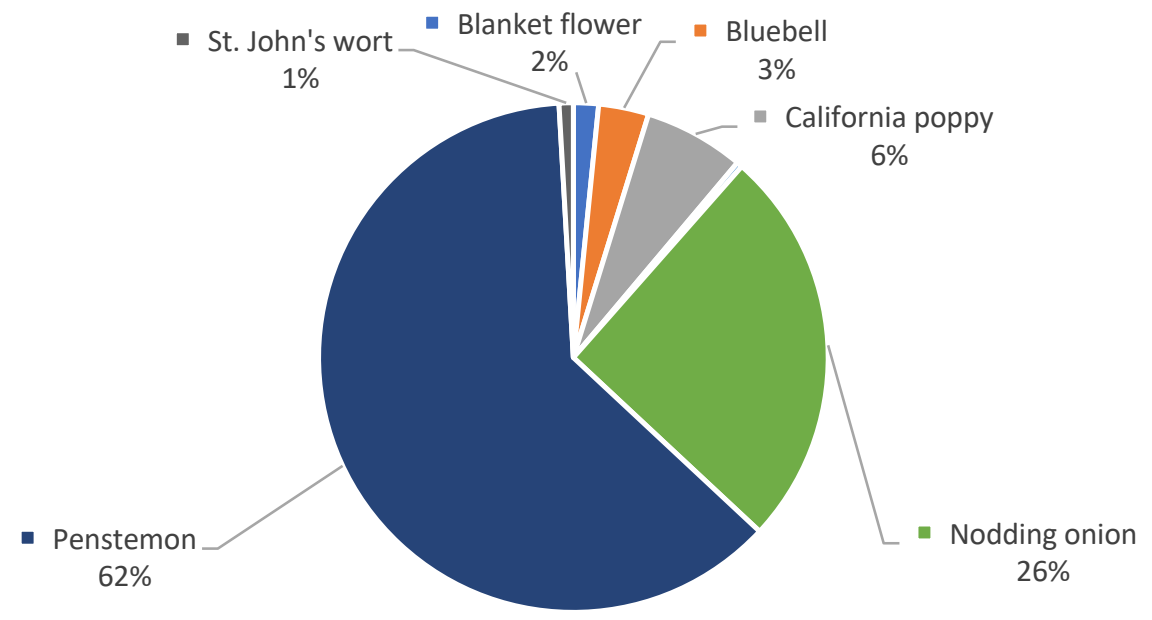

Figure 15. Percent cover floral resource composition at Yukon Bioswales for June. Data collected during vegetation survey. 


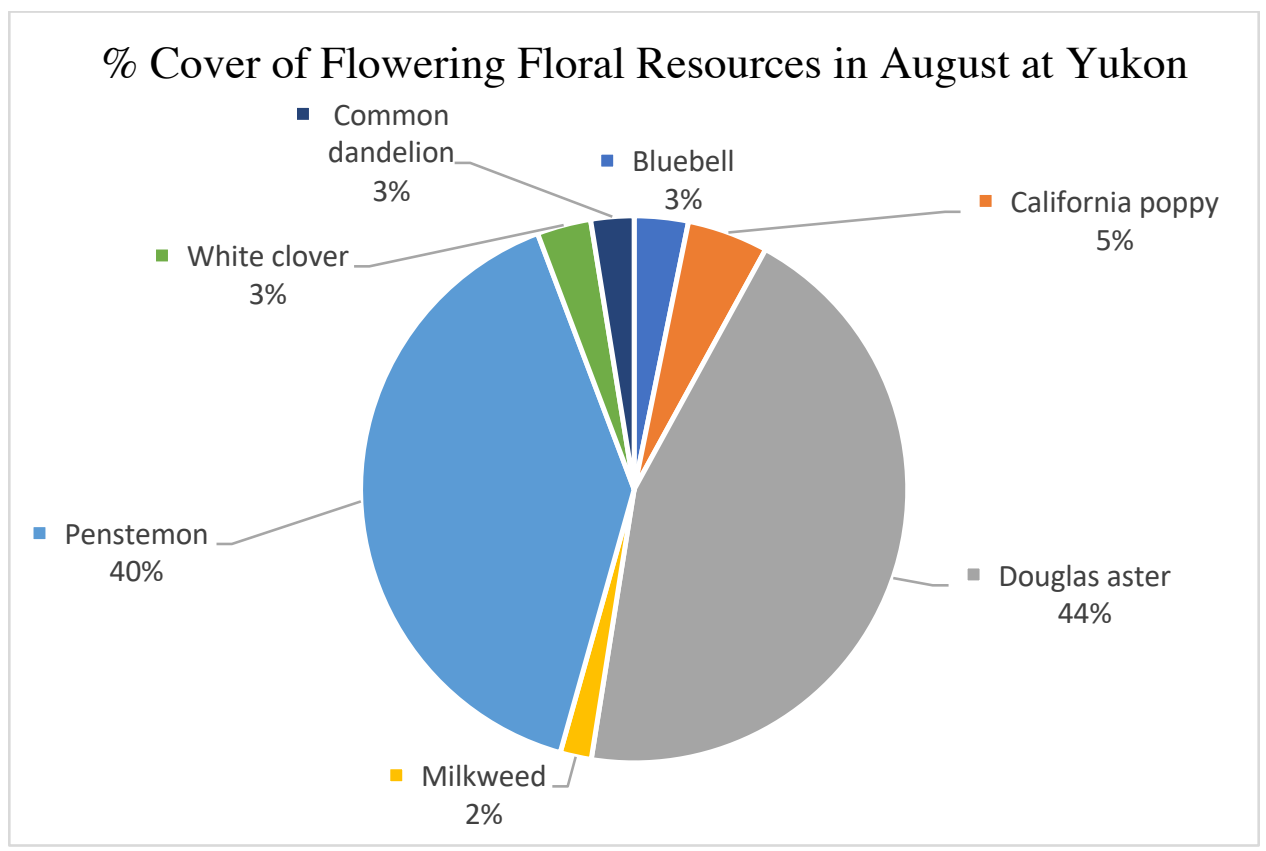

Figure 16. Percent cover of floral resource composition at Yukon Bioswales for August. Data collected during vegetation survey.

\section{Beyer Court}

In June, Beyer Court had the highest floral species richness out of the three sites (Table 3). Floral resource composition was highly variable at Beyer Court in June. Plants had very similar percent cover in the community. Plants that were slightly more predominant at the time included, nodding onion, California poppy and douglas spirea (Figure 17). In July, the species richness at Beyer Court decreased by more than half (Table 3). Floral resource composition shifted very sharply in July with douglas aster becoming the most predominant available flowering resource (Figure 18). In August, floral species richness at Beyer Court again decreased from the following month (Table 3). Beyer Court floral composition in August was not diverse and was predominated by Douglas aster (Figure 19). 


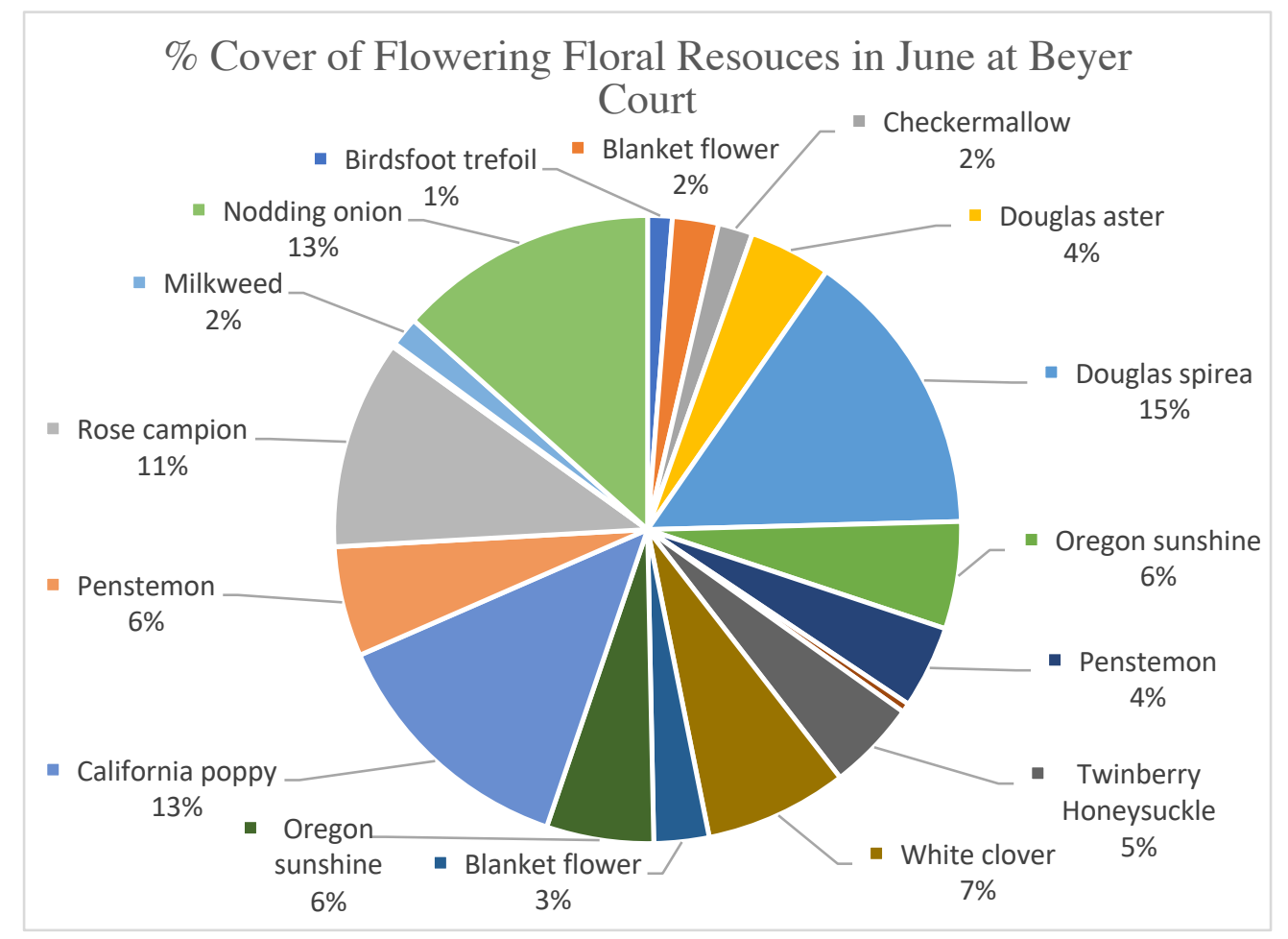

Figure 17. Percent cover floral resource composition at Beyer Court for June. Data collected during vegetation surveys. 


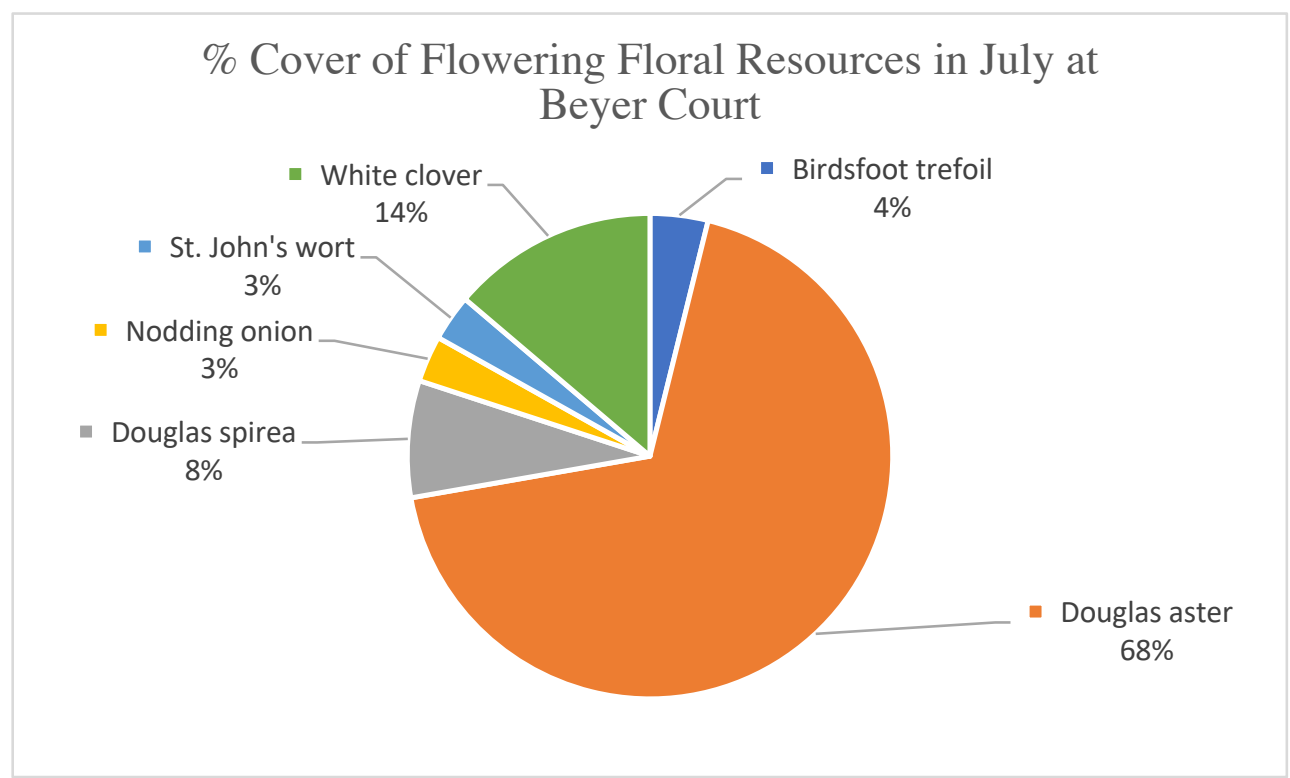

Figure 18. Percent cover floral resource composition at Beyer Court for July. Data collected during vegetation surveys.

$\%$ Cover of Flowering Floral Resources in August at Beyer

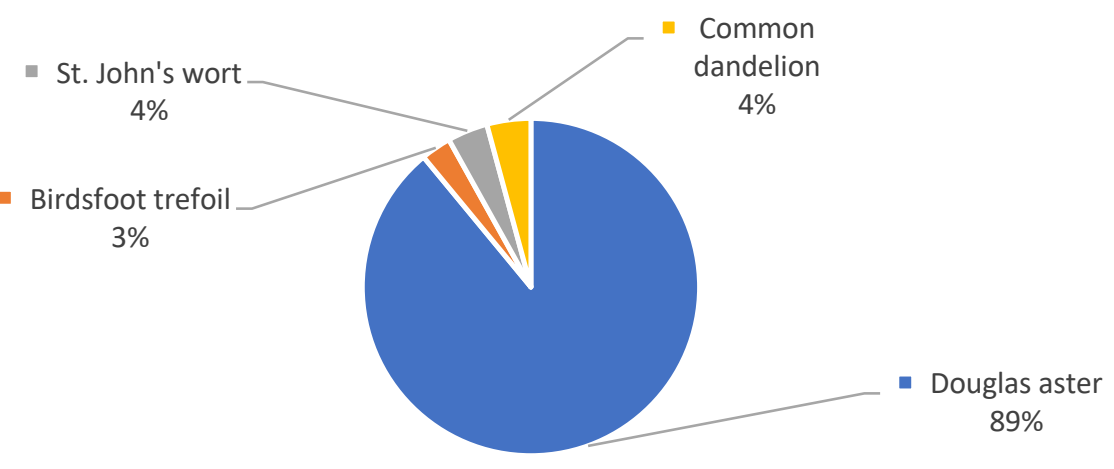

Figure 19. Percent cover floral resource composition at Beyer Court for August. Data collected during vegetation surveys. 


\section{Johnson Creek Commons}

In June, Johnson Creek Commons had the second highest species richness out of the three sites (Table 3). Floral resource composition was highly diverse at this time with California poppy, nodding onion and rose campion being the dominant available resources (Figure 20). In July, the floral species richness at Johnson Creek Commons halved in comparison to June (Table 3). The predominant flowering plants at the site in July were dogwood and Oregon grape (Figure 21). In August, the floral species richness declined further, but predominant floral composition stayed similar to July (Table 3). Predominant flowering plants at Johnson Creek Commons in August included dogwood and Oregon grape (Figure 22).

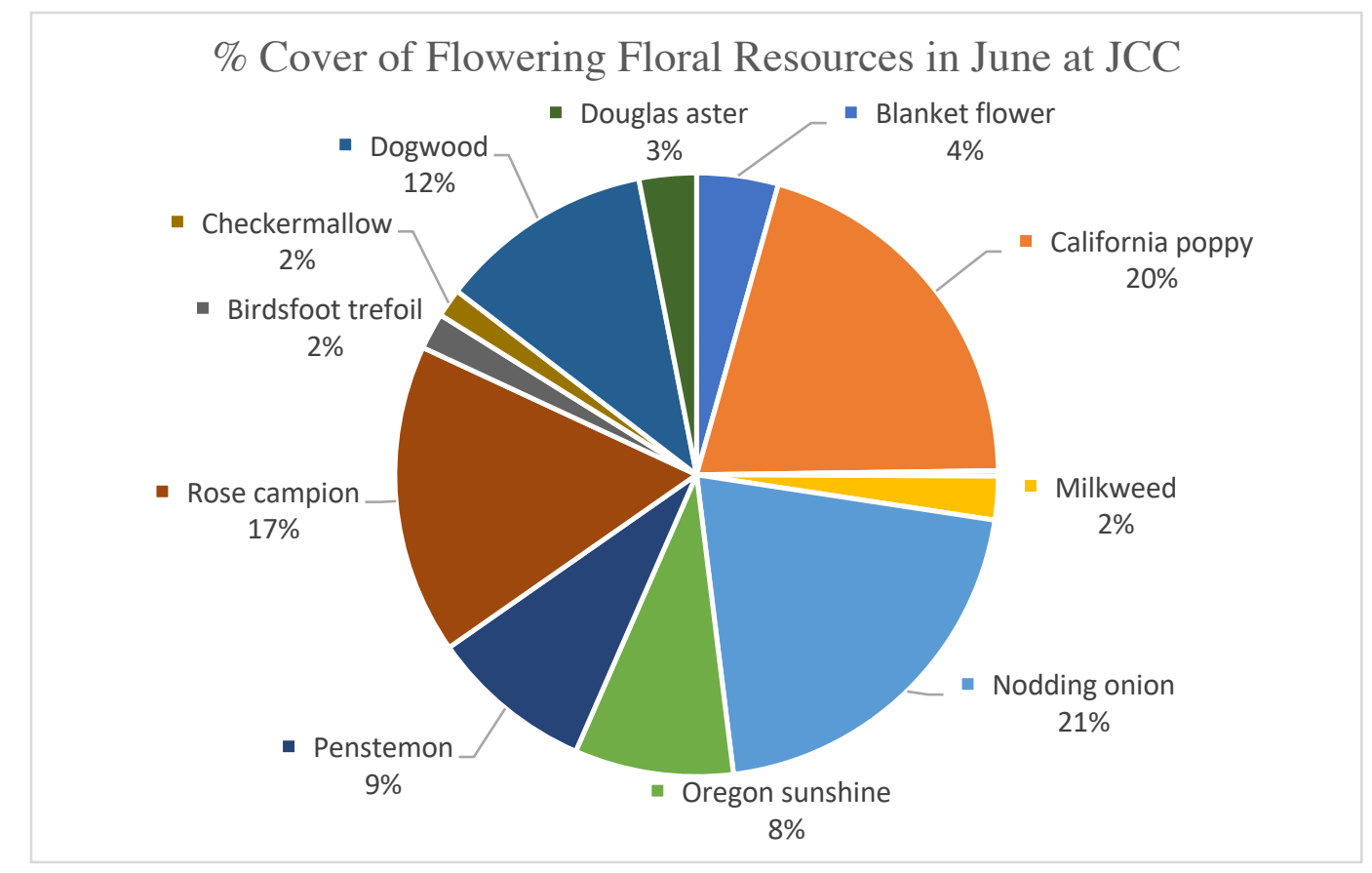

Figure 20. Percent cover floral resource composition at Johnson Creek Commons for July. Data collected during vegetation surveys. 


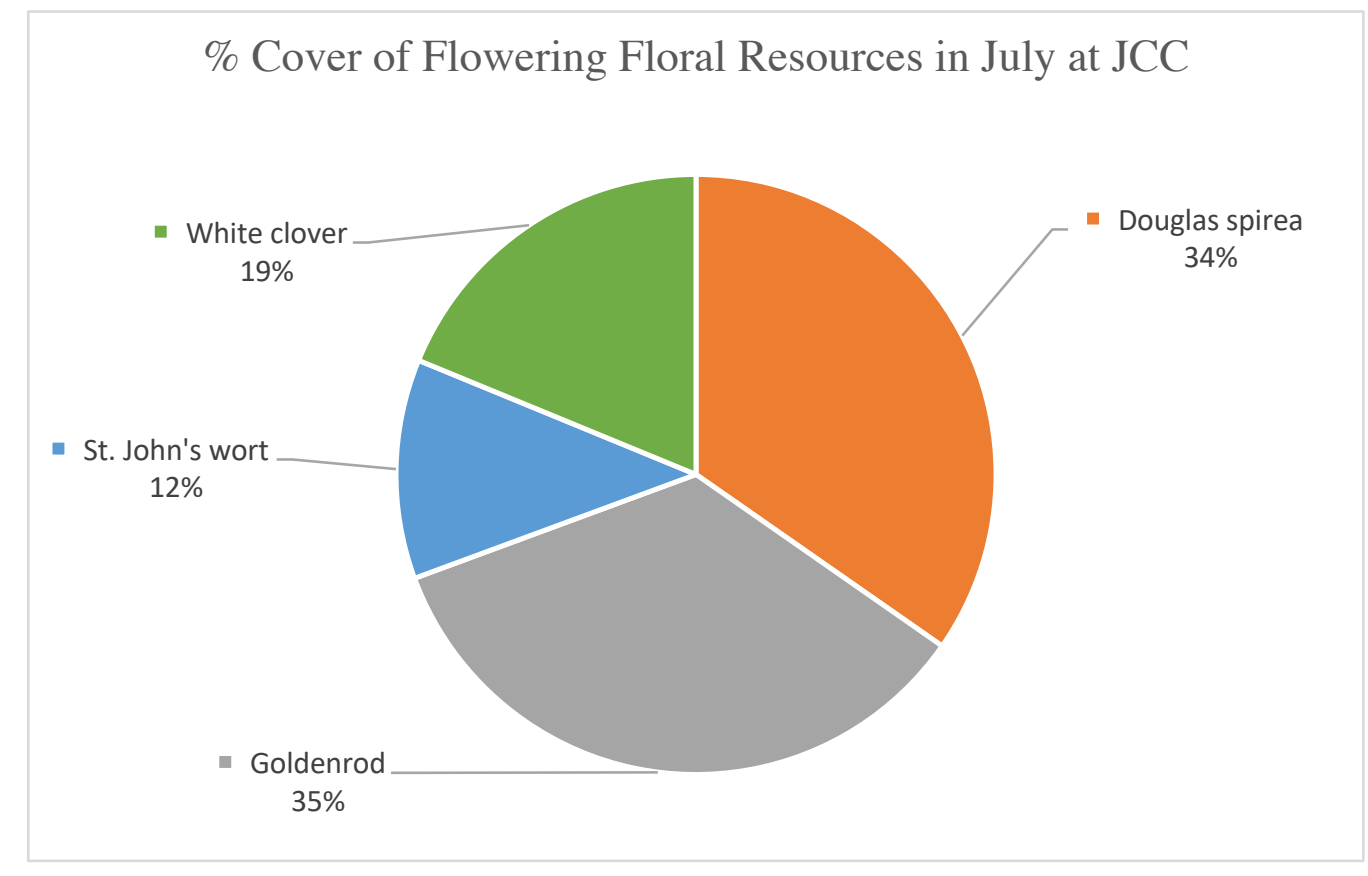

Figure 21. Percent cover floral resource composition at Johnson Creek Commons for July. Data collected during vegetation surveys.

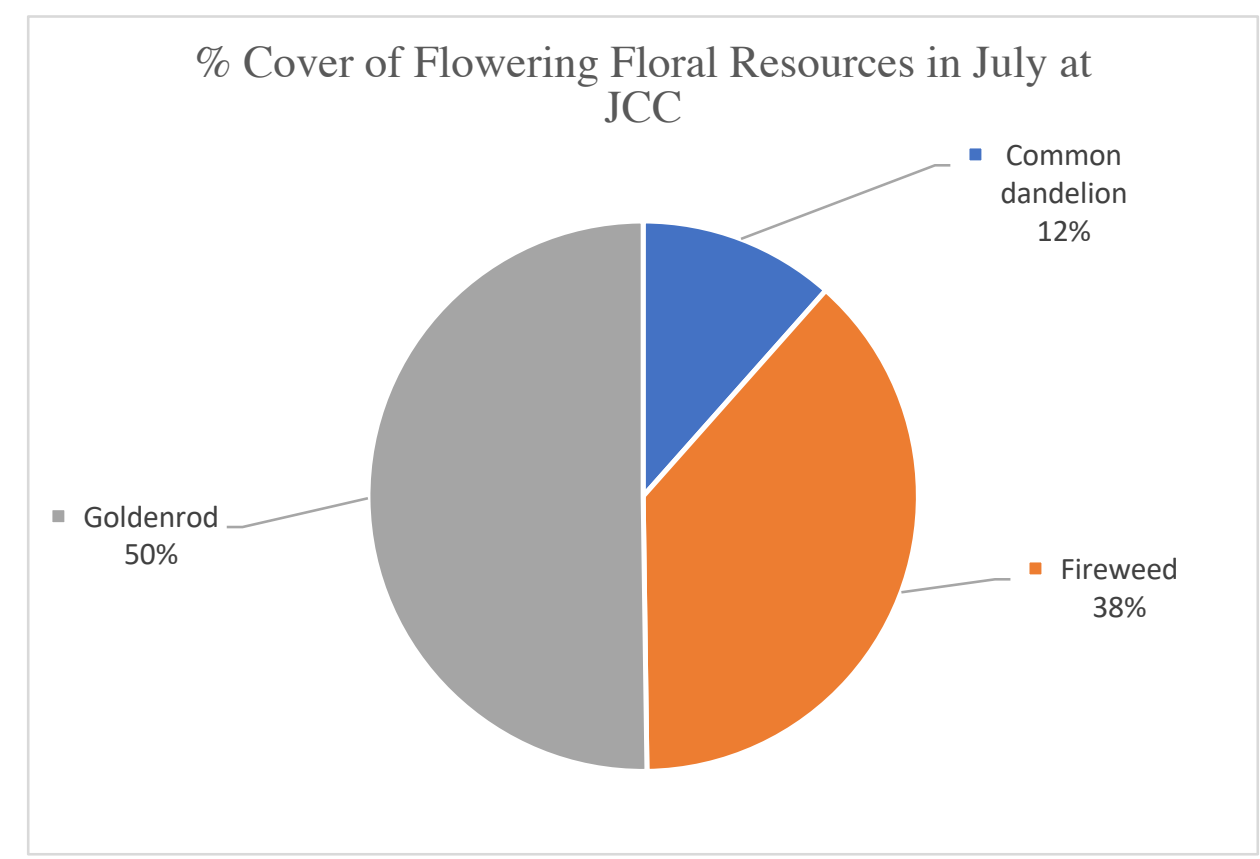

Figure 22. Percent cover floral resource composition at Johnson Creek Commons for August. Data collected during vegetation surveys. 
Native Bee Floral Resource Visitation

Pollinator visitation varied among floral resources with goldenrod having the highest number of bee observations for the entire monitoring season from June to August (Figure 23). Additionally, white clover and dogwood were also frequently visited by native bees. However, floral resources that were thought to be preferred by honey bees and other pollinators such as milkweed had little to no bee observations for the monitoring season at the sites (Figure 23).

Honey Bee Floral Resource Visitation

Honey bee floral resource visitation differed from native bee visitation. Honey bees predominantly visited Penstemon species at the observed sites during the entire monitoring season from June to August. No honey bees were observed on goldenrod or milkweed (Figure 24). In this work, we did find differences between floral resource use in honey bee and native bees. 


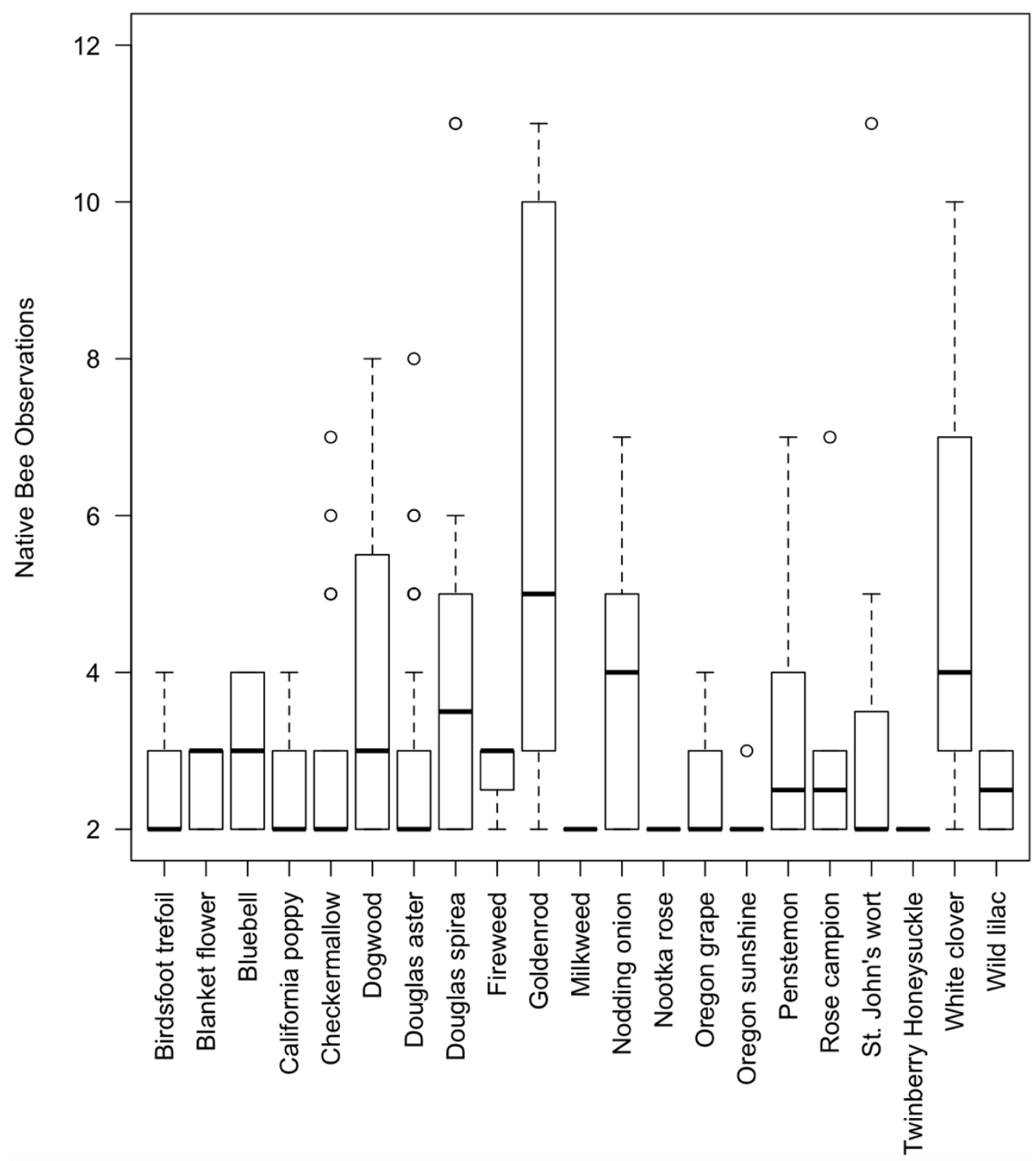

Figure 23. Average Native Bee Floral Resource Visitation from JuneAugust on floral resources at all sites. Dark lines represent the mean number of native bees observed per observational period. The dashed lines represent the maximum number of native bees seen per observational period. The open circles represent outliers. Goldenrod was visited the most frequently, however typical "honey bee preferred plants" such as Milkweed had no native bee observations. 


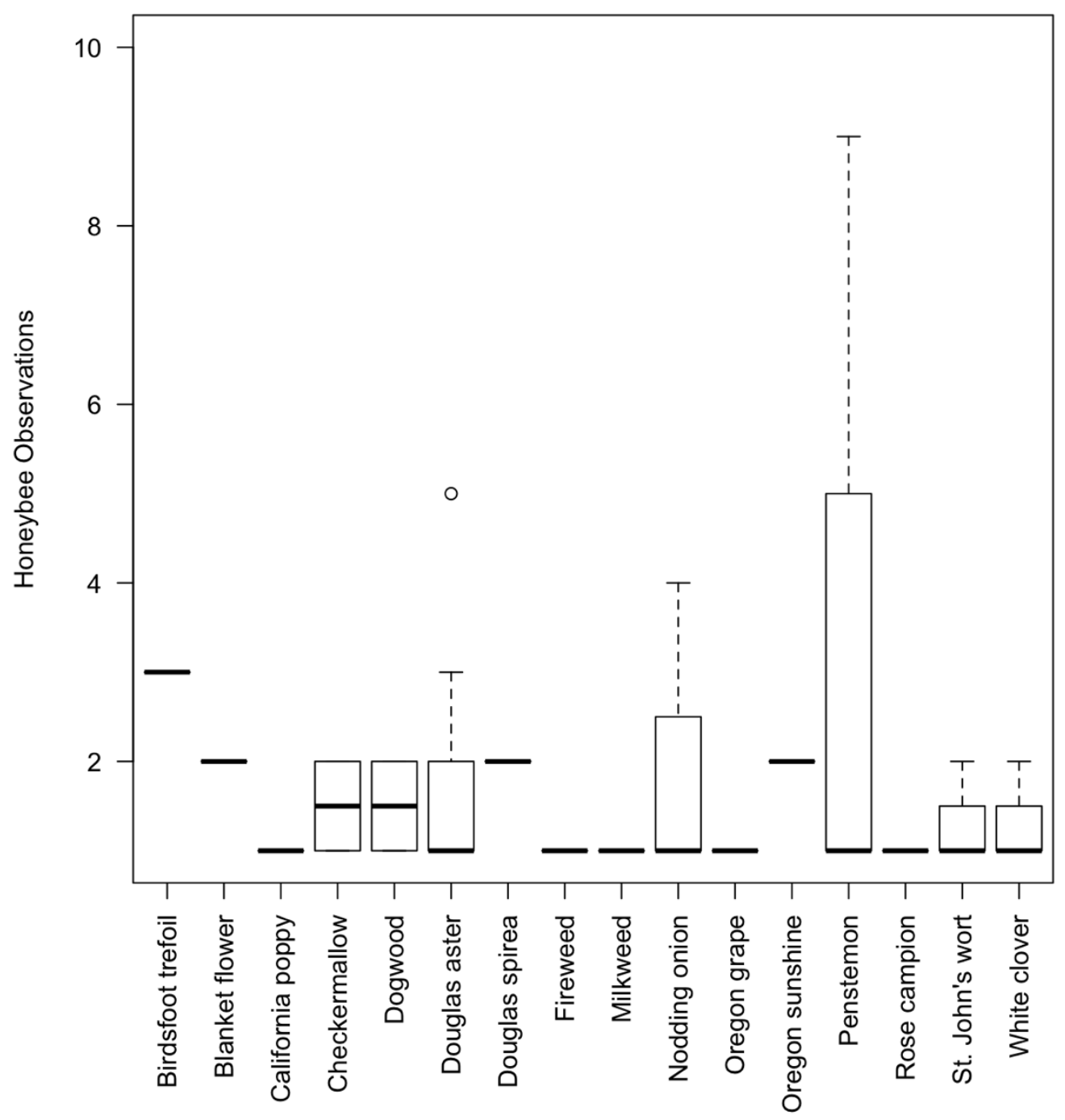

Figure 24. Honey bee Floral Resource Visitation from JuneAugust. Dark lines represent the mean number of honey bees observed per observational period. The dashed lines represent the maximum number of honey bees seen per observational period. The open circles represent outliers. Penstemons were visited the most frequently, however typical "honey bee pollinator plants" such as Milkweed had no observations. 


\section{Honeybees}

Separating native bees and honey bee observations for statistical analysis was not feasible due to the low numbers of honey bees seen throughout the data collection season (Table 4). Due to the low number of honey bees that were seen during vegetation survey days, honey bee observations within two weeks of floral resource vegetation surveys were combined to see if statistical analysis was possible. Combined data was unable to undergo statistical analysis because there were too little observations of honey bees to properly estimate important predictors for presence and fit the complex model. When running the generalized mixed model in $\mathrm{R}$, the model failed to converge due to the lack of data. Due to the inability to run a model on honey bee visitation alone, honey bees were not removed from the data set. Since they were a small portion of bees observed, I presumed that their presence did not heavily influence the hypothesized predictors for all bee visitation.

Table 4. Number of honeybees observed throughout the monitoring season of June, July and August at Beyer Court (BC), Johnson Creek Commons (JCC) and Yukon Bioswales (YBIO).

\begin{tabular}{|l|l|l|l|}
\hline & June & July & August \\
\hline BC & 13 & 8 & 4 \\
\hline JCC & 15 & 12 & 2 \\
\hline YBIO & 14 & 6 & 2 \\
\hline
\end{tabular}

Significant predictors of bee presence

After creating a generalized linear mixed model (GLMM) with Gaussian Hermite Quadrature (GHQ) approximation maximum likelihood five significant variables were identified that predicted bee presence at sites. Significant variables that predicted bee presence included Goldenrod ( $\mathrm{p}$-value 0.024$)$, density of floral resources ( $\mathrm{p}$-value $3 \times 10^{-}$ 
${ }^{6}$ ), the months June (p-value 0.02 ) and July (p-value 0.001 ) as well as tiny dark bees (pvalue 0.0023$)($ Table 5). 
Table 5. Significant variables that predicted bee presence at sites included Goldenrod ( $p$ value 0.024), density of floral resources ( $p$-value 3x10-6), the months June (p-value 0.02) and July (p-value 0.001) as well as tiny dark bees (p-value 0.0023). Significance is illustrated by asterisks.

$\begin{array}{ll}\text { Goldenrod } & \\ \text { P-value } & 0.0024^{* *} \\ \text { Estimate } & (0.445)\end{array}$

The higher the amount of Goldenrod

the more bees were observed

Density

P-value

$0.000003^{* * *}$

Estimate

The higher the density of floral resources, the more bees were observed

June

P-value

$0.002^{* *}$

Estimate

July

P-value

$0.001^{* * *}$

Estimate

Inidcates that more bees were observed in

June and July compared to August

Tiny dark bee

P-value

$0.0023 * *$

Estimate

$(0.365)$

The higher the amount of Tiny Dark Bees that were observed, more bees from other groups were observed

Observations 286

Log Likelihood

$-316.112$

Akaike Inf. Crit.

714.225

Bayesian Inf. Crit.

864.120

Note:

${ }^{*} \mathrm{p}<0.1 ;{ }^{* *} \mathrm{p}<0.05 ;{ }^{* * *} \mathrm{p}<0.01$ 


\section{Other significant relationships}

I was interested in understanding if there were significant differences found between groups in the data. Kruskal-Wallis tests did not find a significant relationship between the number of bee observations and month (p-value 0.574). Additionally, Kruskal-Wallis test did not find a significant relationship observed between bee observations and native or invasive status of floral resources (p-value 0.686 ). Native or invasive status of floral resources was determined by using information from the USDA Plant Profiles. Kruskal-Wallis' tests did disclose a significant difference between bee observation and bee group (p-value 0.008) (Table 6). 
Table 6. A significant difference was found between bee observations and bee group. No significant difference was found between bee observations and native or invasive status of floral resource. No significant difference was found between bee observations and month.

Were there significant differences in bee observations

made between plants that were native or invasive?

Kruskal-Wallis Rank Sum Test

$\mathrm{P}$-value

0.6861

Chi-squared

0.16338

DF

1

No significant difference

Was there a significant difference in observations made between months (June, July, August)?

Kruskal-Wallis Rank Sum Test

P-value

0.574

Chi-squared

1.2913

DF

2

No significant difference

Was there a significant difference in observations made between different bee groups?

Kruskal-Wallis Rank Sum Test

P-value

$0.008^{* * *}$

Chi-squared

23.665

DF

There was a significant difference in observations made between bee groups

Note:

${ }^{*} \mathrm{p}<0.1 ;{ }^{* *} \mathrm{p}<0.05 ;{ }^{* * *} \mathrm{p}<0.01$ 


\section{Discussion}

\section{Urban Bee Composition}

In this work, we found that bee composition was similar at all three sites for the data collection season. Predominant bee groups for the entire data collection season from all sites were tiny dark bees and bumblebees. The tiny dark bee group was composed of individuals from the genera Lasioglossum (sweat bee) and Ceratina (small carpenter bee). Due to the size of individuals in this morphospecies grouping, bees were not split into separate generas due to community science expertise and lack of field microscopes. Tiny dark bees may have been identified as being a bioindicator for other bee groups, meaning, the more tiny dark bees we saw, the more bees in other groups were observed. The elevated presence of tiny dark bees at my sites may have management implications. Their frequency may have indicated that native bees respond better to resources at small spatial scales (Jha et al., 2009) and that it may be more important to provide for tiny dark bees than other bee groups at enhanced pollinator sites in urbanized areas.

The high frequency at which bumblebees and tiny dark bees were observed in a highly urbanized environment and the lack of medium dark bees and metallic hairy belly bees (Osmia) mirrored what we currently understand about bee habitat preference (Collado et al., 2019, Wray et al., 2014).

Collado et al., (2019) characterized bee habitat preference. Bees in the genus Lasioglossum and Bombus were seen as urban adapters which thrived in highly urbanized environments. Bees in the genus Andrena or Osmia were characterized as urban avoiders which preferred forested and non-urbanized habitats. The urban bee composition found in this study in moderately urbanized South East Portland appeared to confirm those characterizations. Urban adaptor tiny dark bees (Lasioglossum and Ceratina) were found 
at $24-37 \%$ of bee composition and urban adaptor bumblebees (Bombus) ranged in abundance from $20-39 \%$ of bee composition. Urban avoider medium dark bees (Andrena), which preferred non-urbanized sites, comprised only $0-3 \%$ of bee composition, and urban avoider metallic hairy belly bees (Osmia) comprised 1-4\% of urban bee composition at these urbanized sites.

Osmia and Andrena were identified in this study as being urban avoiders as well as in other research (Collado et al., 2019). However, Osmia and Andrena are quite common around Portland in the early spring (Susan Masta, personal communication). The monitoring time for this project starting in June did not adequately sample early emerging bees such as Osmia and Andrena. Although not observed at our sites, populations of Osmia and Andrena are common around Portland. It is also important to note the limitations of using the Xerces' Maritime monitoring guide for citizen scientists (Minnerath et al., 2014). Because the protocol used morphospecies categories, multiple genera were combined into a single grouping such as the group "Tiny Dark Bee" which contained individuals from the genus Lasioglossum and Ceratina. This morphospecies grouping did not allow for the identification of individual genera but was the easiest to use with community members. Although some specification was lost, general trends of bee visitation were still observable.

\section{Floral Resource Composition}

The sharp decline in species richness across all sites from June to August illustrated the lack of late season floral resources at these sites. Although plants at each site varied considerably, the total number of bees observed were similar for all three sites. Although month (June and July) was an important predictor for bee presence, there was not a significant difference between observations and months (June, July, August). This 
indicated that the floral resources available in June and July were more plentiful than those available in August. Floral resource composition at sites could have explained why June and July were identified as significant predictors for bee observations due to the large variety of floral resources available to bees during that time frame as illustrated by the floral richness of sites. August was not identified as a significant predictor due to the lack of floral resources available during that month, however, the number of bee observations was not significantly different between the three months. This may have indicated that if sites had diverse late blooming plants, there would have been a higher rate of bee observations in August than what we observed.

There was a sharp contrast between plant composition at sites in June compared to August. For example, at Johnson Creek Commons in June, there were five times more flowering floral resources than found during August at the same site. The huge floral resource shift in three months greatly limited bee foraging opportunities due to the lack of late-season blooming plants available to bees at sites. Sites for this monitoring project were of similar size composition but, floral resource composition at each site varied, however, the number of bee observations remained similar between all sites for the data collection season. Additionally, there were a few days in August that had smoky and hazy conditions from fires in the area. Monitoring conducted during this time frame resulted in observing very few bees. Floral resource availability as well as weather conditions may have limited our bee observations at sites in August.

With limited floral resources in August, bees did not have the option to be "picky" about what floral resource they were foraging on, when there were only a few flowering resources available. However, we did not observe decreased bee observations during the month of August even though flowering floral resources were very limited 
compared to earlier months (Figure 25, 26, 27). This finding suggested that there were just as many bee pollinators active during the late bee season, compared to the midseason where plentiful floral resources were available. It is highly likely with the addition of late season blooming plants, our sites would reflect a higher number of bee observations in August than what we observed.

Not surprisingly, the few late season blooming plants that were available at sites had a high number of bee observations such as Solidago canadenisis. Although, the floral resource itself was not a large proportion of flowering plant cover at all sites, the proposed significance may be due to the lack of any other late blooming floral resources at sites.

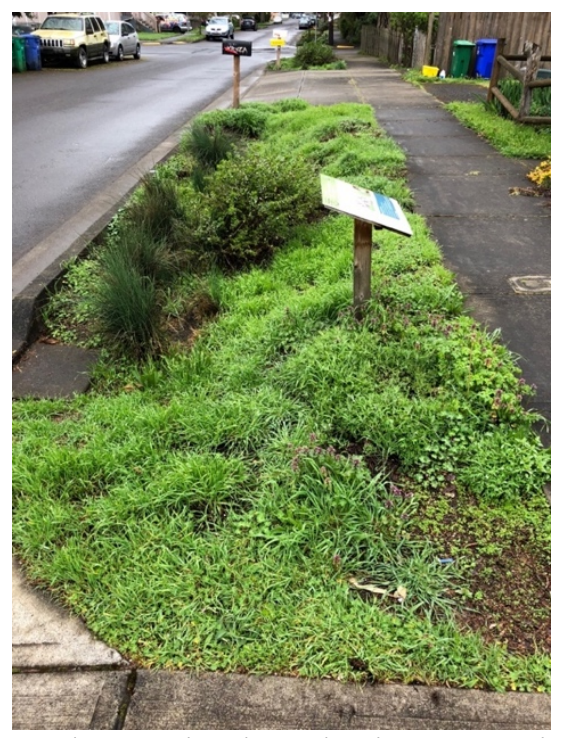

Figure 25. Yukon Bioswale monitoring site in May prior to monitoring occurring. Site had full vegetation and numerous flowering plants. 


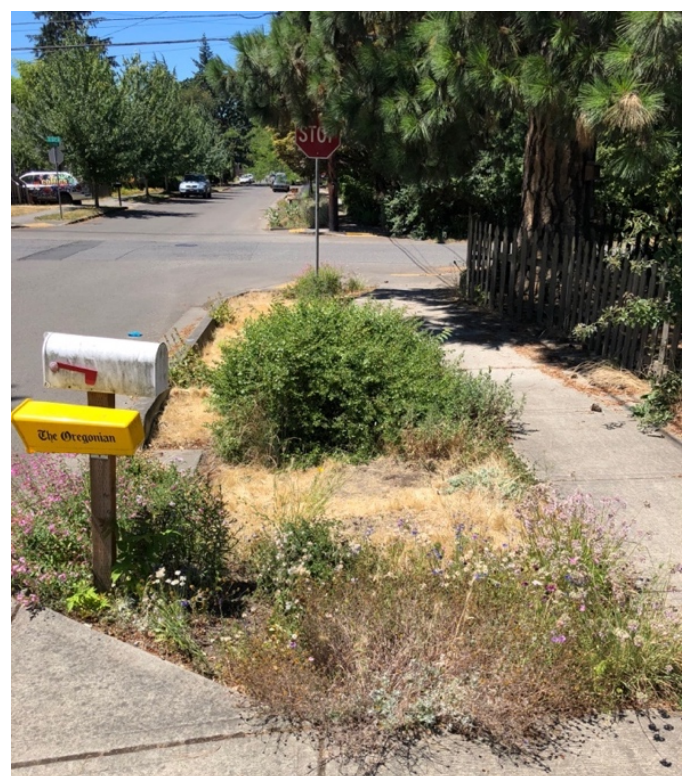

Figure 26. Yukon Bioswale monitoring site in Mid July. Site was very dry and had greatly decreased floral diversity.

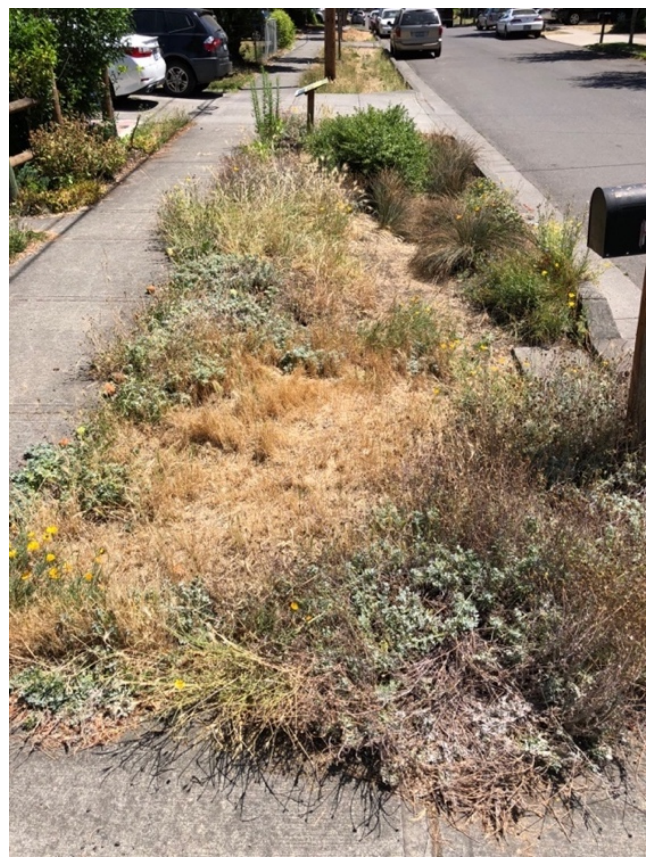

Figure 27. Yukon Bioswale monitoring site in August. Site was extremely dry and had very few flowering resources. 


\section{Native or invasive status}

Native or invasive status of floral resources relating to bee observations has been studied previously and has had contrasting results (Tepedino et al., 2008; Nienhuis et al., 2009). Habitat composition seems to play a large role in invasive floral resource visitation. In this work, native or invasive floral resource preferences of bees were tested, and bee observations did not differ between plants with either invasive or native status. Although statistically there was not a difference between plant status and bee observation, there are clear patterns of floral resource preference in our monitoring results.

Invasive plants have similar characteristics to weeds such as being prolific and persistent which makes them successful invaders into a healthy ecosystem. Plants with weedy growth patterns such as dandelions have been shown to have the highest diversity of bee visitors compared to non-weedy plants (Tommasi et al., 2004). The idea that native bees prefer "weedy plants" was mirrored in our results with native bees preferring goldenrod, St. John's wort, birdsfoot trefoil and white clover. However, native bees visited both native and invasive "weedy species". Honey bee preferences differed from weedy preferences of native bees, as honey bees preferred douglas aster, checkermallows, and penstemons which are all native to the Pacific North West. In our results, honey bees did not prefer "weedy species" such as goldenrod that native bees did. However, a few honey bees were observed on invasive floral resources such as St. John's wort. Interestingly, it is thought that honey bee pollination contributed very little to the success of most weedy species (Huryn 1997) indicating that native bees may play a specialized role in weed pollination. In our results, floral resource characteristics seemed to be more important than native or invasive status for bee visitation. However, this perceived 
importance may be due to the lack of late season floral resources that were available at sites, leading bees to be less "picky" between native or invasive plants for resources. Floral Resource Visitation

Native Bees

Native bees included all morphospecies bee groups found in Portland except honeybees. Native bee visitation for the entire data collection season indicated that goldenrod was the most highly visited plant. In addition, white clover and dogwood were both visited frequently. Native bee floral resource observations greatly differed from preferred resources of honeybees. It's important to separate honeybee and native bee floral preferences in order to correctly manage for native bees. As illustrated by our observational data, honeybees were a very small percentage of our total bees observed. With the recent decline of honeybees associated with Colony Collapse Disorder (CCD), ridovirus and microsporidian (Bromenshenk et al., 2010) native bees can be a great free alternative, and pollinator habitat in urbanized areas should focus on providing resources for native bees.

Honey Bees

Honeybee visitation for the entire data collection season illustrated that the preferred floral resources for honeybees were Penstemon spp. Interestingly, the preferred floral resource of native bees, goldenrod, was not visited by any honeybees during our data collection season. Additionally, typical "pollinator plants" such as milkweed had no honey bee or native bee observations. It's important to keep in mind that these results are site specific, as the bees may have utilized another resource over milkweed at the study sites. 
Based on these results, I conclude that we need to re-think which plants we've identified as being "pollinator plants" especially at a small-scale resource level in urbanized environments. Foraging behaviors exhibited by honey bees were quite different than observed behavior exhibited by native bees. It has been suggested that honeybees preferred large foraging scales with highly productive and mass flowering sites (Jha et al., 2009). In our results, honey bees were not observed on weedy or single patches of floral resources, instead they utilized large patches of closely planted Penstemon spp. which made up 39-41\% of floral resource composition at sites. In comparison, native bees were found to utilize goldenrod which made up 8-9\% of floral resource composition.

These findings illustrate the different foraging behaviors of native and honey bees observed in our monitoring efforts which is important for managing enhanced pollinator sites. Additionally, with findings from this work, I was able to make recommendations to management on which specific floral resources to plant that provide for native bees or honeybees. These recommendations can be found in the "Implications to Management" sections of this thesis.

What predicted bee presence at sites in South East Portland?

\section{Goldenrod}

Goldenrod was one of the few late blooming floral resources at sites, which may have indicated why it was found to be a significant factor that explained bee presence. The higher the amount of Goldenrod that was at sites, the more bees were observed. The predicted significance may be due mostly to the floral bloom time of goldenrod, in addition to its frequency at sites late during the season. It's worth noting that in our data collection we did not observe any honey bees utilizing goldenrod, however, it had a high frequency of native bee use. Goldenrod is a Pacific Northwest native and it has recently 
been found that it has a high attractiveness to native bees in urbanized areas, as well as in more rural areas such as Corvallis, Oregon (Aaron Anderson, Personal Communication). It's important to note here that the predicted importance of goldenrod may be due to the fact that it was one of the few late season blooming resources, and although it did not make up a large composition of floral resources at all sites, it was one of the few available resources in late July and August at Johnson Creek Commons.

\section{Habitat composition}

Habitat composition had a very strong effect on bee composition on a small community scale. It has been found that bees foraging responses depend on spatial scale and that habitat composition had a stronger effect than distance to the nearest forest fragment (Jha et al., 2009). I had hypothesized that inflorescence blossom density of individual floral resources would be an important predictor of bee presence due to the idea that small scale floral resources are extremely important for bee survival. However, inflorescence blossom density was not a significant predictor of bee presence, but instead we found that the higher the density of all floral resources at a site, the more bees were observed. The importance of dense floral resources to bees has been shown previously as bees appear to act as optimal foragers on a small community scale because they are more heavily influenced by floral density than floral diversity (Hegland \& Boeke 2006).

This study also suggested that small bodied bees, such as tiny dark bees, respond to resources at a small spatial pattern in contrast to honeybees which spread out between high resource patches illustrated by the high Penstemon spp. honeybee visitation rate. My results indicated that tiny dark bees may have served as bioindicators. This is supported by other studies showing that native bees respond better to resources at small spatial scales (Jha et al., 2009). 


\section{Implications to management}

Urban areas provide the opportunity to aide native bees by providing floral resources and site care in the built environment. From this work, I have identified three main areas of concern for pollinator habitat management in South East Portland; late season plants, specific pollinator plant lists and site care.

\section{Adding late season plants}

Late season floral resources were not available at sites in August indicated by the sharp decline in floral species richness. Many bees rely on late season plants to provide nectar and pollen resources. Designated pollinator areas, especially in cities have the potential to attract diverse seasonal native bee taxa if diverse floral resources are provided throughout the growing season (Wojcik et al., 2008). I recommend adding late season blooming plants to established pollinator habitat such as goldenrod for native bee use and Penstemon spp. for honey bee use. At our site, goldenrod bloomed July-August and was one of the only late blooming plants at Johnson Creek Commons besides common dandelions. Adding late season plants could also breakup the resources at these small sites, so they are more attractive to native bees than honeybees (Jha et al., 2009).

\section{Specific pollinator plant lists}

From this research, identification of the preferred use of floral resources by native bees or honey bees was possible. This allowed for the creation of specific pollinator plant lists. These lists can be used to plant pollinator areas with specific floral resources that were utilized by bees in South East Portland.

The top five floral resources that should be planted into native bee pollinator habitat were (in order of the most utilized): Solidago canadenisis, Trifolium repens, 
Cornus sericea, Allium cernuum and Penstemon spp. Pollinator habitat can also be planted with specific floral resources most utilized by honey bees. Including (in order of the most utilized): Penstemon spp., Allium cernuum, Aster subspicatus and Lotus corniculatus. Additionally, mixed honey bee and native bee preferred use floral resource patches can be created by using a mix of utilized floral resources for both groups. For example, by planting: Solidago canadenisis, Penstemon spp., and Allium cernuum, these floral resources would provide for both native bees and honey bees.

\section{Establishing site care}

At the Yukon Bioswales sites, it was clear that homeowners in the area had planted their own preferred plants in the bioswales. This was evident at almost all sites when comparing the "pollinator plant lists" that were utilized by Green Lents while planting the sites, compared to the actual plants that were established at the sites. These plants, such as rose campion were not frequently visited by bees. Homeowners adding plants into bioswales and designated pollinator habitat can become a large issue when the plant that isn't preferred by the bee is taking up crucial space for a preferred plant that provides resources.

Designated pollinator habitats require adaptive management in order to be successful and provide resources for native bees. Weekly site care should be invested such as hand watering or weeding out invasive species. Our monitoring sites were not irrigated and to my knowledge were never watered by hand. Due to this, in August, all sites were extremely dry which limited the available floral resources. This is an issue when late season floral resources are so crucial for bees. Additionally, pollinator habitats should not be mowed, and plants should not be cut down during the high resource season, 
after summer is over bare dirt and leaves should be left to provide overwintering sites for bumblebee queens.

Community Science

The aim of this project was to involve as many community members as possible to learn about Oregon's native pollinators and monitor pollinator sites. However, this did not happen as I ended up training 6 individuals, 4 of whom collected data for the observational season. Due to the low number of total participants survey questionnaires were not utilized but relaxed discussions about involvement post project were conducted. Those volunteers who were involved with BeeCountPDX were grateful for their involvement, and one volunteer was so interested about doing bee collections that I got her involved with the Oregon Bee Atlas. Individuals who were involved in the project were excited to learn about native bees and monitor them for the summer.

Community science volunteers collected about twenty percent of the observational data, I collected the other eighty percent. The volunteers were trained to identify morphospecies and were encouraged to take photos while they were monitoring if they were unable to identify a bee, I would then verify the conclusion they came to from the photos. When volunteer observations were unsure and sent to myself, they were correct $95 \%$ of the time. The high rate of successfully identified bee groups by volunteers assured me of the quality of data being collected. 


\section{Conclusion}

In this work, we found that bee composition was similar at all three sites for the data collection season. Predominant bee groups for the entire data collection season from all sites were tiny dark bees and bumblebees. The high frequency at which bumblebees and tiny dark bees were observed in a highly urbanized environment and the lack of medium dark bees and metallic hairy belly bees (Osmia) mirrored what we currently understand about bee habitat preference (Collado et al., 2019, Wray et al., 2014).

Floral resource composition variation at sites could have explained why June and July were identified as significant predictors for bee observations due to the large variety of floral resources available to bees during that time frame. August was not identified as a significant predictor due to the lack of floral resources available during that month. These results indicated the lack of late season flowering resources at sites. Additionally, blossom density was not identified as being a significant predictor of bee presence. This observation may have indicated that bees did not have the option to be "picky" about what floral resource they were gathering resources from, when there were only a few flowering resources available. It is also possible that with the addition of late blooming plants at enhanced sites in South East Portland there could be increased bee observations during the late bee season.

In our results, honey bees and native bees had different floral resource preferences. Honey bees were not observed on weedy or single patches of floral resources, instead they preferred large patches of closely planted Penstemons spp. which made up 39-41\% of floral resource composition at sites. In comparison, native bees were found on small patches and weedy species and were found to prefer goldenrod which made up $8-9 \%$ of floral resource composition at sites. Additionally, native or invasive 
status of floral resources was not significant when related to bee visitation. These findings illustrate the different foraging behaviors of native and honey bees observed which is important for managing enhanced pollinator sites. Additionally, these findings indicate preferred resources for native and honey bees, allowing for the planting of specific honey bee or native bee pollinator habitat in South East Portland. Bee pollinators can thrive in highly urbanized environments if their preferred floral resources and habitat types are available.

\section{Future work}

In urbanized environments it is important to manage for urban adapted species such as bumblebees and tiny dark bees, by providing habitat and preferred resources. Future research into small scale habitat preferences of native bees is needed. Research similar to this thesis work needs to be done for all urbanized environments, as results from this monitoring experiment are specific to South East Portland. With increasing urbanization, land use change and population growth, cities have the responsibly to try and help native bee populations by implementing small changes into enhanced pollinator site construction.

It would be ideal to set up a community science monitoring program involving residents who live in or near ROSE CDC's housing, right outside the enhanced sites. This would allow for the spread of scientific literacy and would benefit those who are living near these sites socially as well as mentally. Future pollinator monitoring should occur at these enhanced sites to observe the shift in bee communities with the further establishment of floral resources at pollinator enhanced sites. Pollinator monitoring should also occur at newly enhanced sites that Green Lents is constructing. Additionally, results and recommendations from this work should be included in future enhanced 
pollinator site construction, or even incorporate new management techniques with existing sites. 


\section{References}

Bates, D., Maechler, M., Bolker, B., Walker, S., (2015). Fitting Linear Mixed-Effects Models Using lme4. Journal of Statistical Software, 67(1), 1-48. doi:10.18637/jss.v067.i01.

Baxter, J. (2014). Vegetation Sampling Using the Quadrat Method [Class handout]. Department of Biological Sciences, Sacramento State, Sacramento, CA.

Beninde, J., Veith, M., \& Hochkirch, A. (2015). Biodiversity in cities needs space: a meta-analysis of factors determining intra-urban biodiversity variation. Ecology letters, 18(6), 581-592.

Bolker, B. M., Brooks, M. E., Clark, C. J., Geange, S. W., Poulsen, J. R., Stevens, M. H. H., \& White, J. S. S. (2009). Generalized linear mixed models: a practical guide for ecology and evolution. Trends in ecology \& evolution, 24(3), 127-135.

Breslow, N.E. (2004) Whither PQL? In Proceedings of the Second Seattle Symposium in Biostatistics: Analysis of Correlated Data (Lin, D.Y. and Heagerty, P.J., eds), pp. 1-22, Springer.

Bretagnolle, V., \& Gaba, S. (2015). Weeds for bees? A review. Agronomy for Sustainable Development, 35(3), 891-909.

Bromenshenk, J. J., Henderson, C. B., Wick, C. H., Stanford, M. F., Zulich, A. W., Jabbour, R. E., \& Williams, T. (2010). Iridovirus and microsporidian linked to honey bee colony decline. PloS one, 5(10), e13181.

Burnham, K. P., \& Anderson, D. R. (2004). Multimodel inference: understanding AIC and BIC in model selection. Sociological methods \& research, 33(2), 261-304.

Cane, J. H. (1991). Soils of ground-nesting bees (Hymenoptera: Apoidea): texture, moisture, cell depth and climate. Journal of the Kansas Entomological Society, 406-413.

Cannon, W. F., \& Horton, J. D. (2009). Soil geochemical signature of urbanization and industrialization-Chicago, Illinois, USA. Applied Geochemistry, 24(8), 15901601 .

Chmielewski, F. M., Müller, A., \& Bruns, E. (2004). Climate changes and trends in phenology of fruit trees and field crops in Germany, 1961-2000. Agricultural and Forest Meteorology, 121(1-2), 69-78.

Collado, M. Á., Sol, D., \& Bartomeus, I. (2018). Bees use anthropogenic habitats despite strong natural habitat preferences. Diversity and Distributions, 2019;1-12. 
Cook, S. M., Awmack, C. S., Murray, D. A., \& Williams, I. H. (2003). Are honey bees' foraging preferences affected by pollen amino acid composition?. Ecological Entomology, 28(5), 622-627.

Deepayan, S., (2008) Lattice: Multivariate Data Visualization with R. Springer, New York. ISBN 978-0-387-75968-5

De Menezes Pedro, S. R., \& De Camargo, J. M. F. (1991). Interactions on floral resources between the Africanized honey bee Apis mellifera $\mathrm{L}$ and the native bee community (Hymenoptera: Apoidea) in a natural" cerrado" ecosystem in southeast Brazil. Apidologie, 22(4), 397-415.

Greenleaf, S. S., Williams, N. M., Winfree, R., \& Kremen, C. (2007). Bee foraging ranges and their relationship to body size. Oecologia, 153(3), 589-596.

Harrison, X. A. (2014). Using observation-level random effects to model overdispersion in count data in ecology and evolution. PeerJ, 2, e616

Hawkins, J., de Vere, N., Griffith, A., Ford, C. R., Allainguillaume, J., Hegarty, M. J., ... \& Adams-Groom, B. (2015). Using DNA metabarcoding to identify the floral composition of honey: A new tool for investigating honey bee foraging preferences. PLoS One, 10(8), e0134735.

Hernandez, J., G. Frankie, and R. Thorp. 2009. Ecology of Urban Bees: A Review of Current Knowledge and Directions for Future Study. Cities and the Environment (CATE) 2.

Hegland, S. J., \& Boeke, L. (2006). Relationships between the density and diversity of floral resources and flower visitor activity in a temperate grassland community. Ecological Entomology, 31(5), 532-538.

Huryn, V. M. B. (1997). Ecological impacts of introduced honey bees. The quarterly review of biology, 72(3), 275-297.

Jha, S., \& Kremen, C. (2013). Resource diversity and landscape-level homogeneity drive native bee foraging. Proceedings of the National Academy of Sciences, 110(2), $555-558$

Minnerath, A., M. Vaughan, E. Mader. 2014. Maritime Northwest Citizen Science Monitoring Guide for Bees and Butterflies. 2nd Edition. 60 pp. Portland, Ore: The Xerces Society.

Nienhuis, C. M., Dietzsch, A. C., \& Stout, J. C. (2009). The impacts of an invasive alien plant and its removal on native bees. Apidologie, 40(4), 450-463. 
Osborne, J. W. (2010). Improving your data transformations: Applying the Box-Cox transformation. Practical Assessment, Research \& Evaluation, 15(12), 1-9.

Pinho, L. G. B., Nobre, J. S., \& Singer, J. M. (2015). Cook's distance for generalized linear mixed models. Computational Statistics \& Data Analysis, 82, 126-136.

Podobnik, B. (2011). Assessing the social and environmental achievements of New Urbanism: evidence from Portland, Oregon. Journal of Urbanism: International Research on Placemaking and Urban Sustainability, 4(2), 105-126.

Ramalho, M., Kleinert-Giovannini, A., \& Imperatriz-Fonseca, V. L. (1990). Important bee plants for stingless bees (Melipona and Trigonini) and Africanized honeybees (Apis mellifera) in neotropical habitats: a review. Apidologie, 21(5), 469-488.

R Core Team (2017). R: A language and environment for statistical computing. R

Foundation for Statistical Computing, Vienna, Austria. URL https:/www.Rproject.org/.

Ritcher, P. O. (1966). Biological control of insects and weeds in Oregon.

Tepedino, V. J., Bradley, B. A., \& Griswold, T. L. (2008). Might flowers of invasive plants increase native bee carrying capacity? Intimations from Capitol Reef National Park, Utah. Natural Areas Journal, 28(1), 44-51.

Thomson, D. (2004). Competitive interactions between the invasive European honey bee and native bumble bees. Ecology, 85(2), 458-470.

Tommasi, D., Miro, A., Higo, H. A., \& Winston, M. L. (2004). Bee diversity and abundance in an urban setting. The Canadian Entomologist, 136(6), 851-869.

Vanbergen, Adam J., and the Insect Pollinators Initiative. "Threats to an ecosystem service: pressures on pollinators." Frontiers in Ecology and the Environment 11.5 (2013): 251-259.

Vargha, A., \& Delaney, H. D. (1998). The Kruskal-Wallis test and stochastic homogeneity. Journal of Educational and Behavioral Statistics, 23(2), 170-192.

Venables, W. N. \& Ripley, B. D. (2002) Modern Applied Statistics with S. Fourth Edition. Springer, New York. ISBN 0-387-95457-0

Wickham, H., François, R., Henry, L., and Müller, K., (2018). dplyr: A Grammar of Data Manipulation. R package version 0.7.8. https://CRAN.Rproject.org/package $=$ dplyr

Winfree, R., Williams, N. M., Dushoff, J. and Kremen, C. (2007), Native bees provide insurance against ongoing honey bee losses. Ecology Letters, 10: 1105-1113. doi:10.1111/j.1461-0248.2007.01110.x 
Wojcik, V. A., Frankie, G. W., Thorp, R. W., \& Hernandez, J. L. (2008). Seasonality in bees and their floral resource plants at a constructed urban bee habitat in Berkeley, California. Journal of the Kansas Entomological Society, 15-28.

Wray, J. C., Neame, L. A., \& Elle, E. (2014). Floral resources, body size, and surrounding landscape influence bee community assemblages in oak-savannah fragments. Ecological Entomology, 39(1), 83-93. 


\section{Appendices}

Appendix A. List of plants that were initially planted by Green Lents at Johnson Creek Commons, Beyer Court Rain Garden and Yukon Bioswale enhanced pollinator sites.

\begin{tabular}{|l|l|}
\hline \multicolumn{2}{|c|}{ Johnson Creek Commons } \\
\hline Large leaved lupine & \\
\hline Common rush & Lupinus polyphyllus \\
\hline Spreading rush & Juncus effusus \\
\hline Goldenrod & Juncus patens \\
\hline Beach daisy & Solidago canadensis \\
\hline Great camas & Erigeron glaucus \\
\hline Checkermallow & Camassia leichtlinii \\
\hline Idaho fescue & Sidalcea spp. \\
\hline Pacific ninebark & Festuca idahoensis \\
\hline Nootka rose & Physocarpus capitatus \\
\hline Red maple & Rosa nutkana \\
\hline
\end{tabular}

\begin{tabular}{|l|l|}
\hline \multicolumn{2}{|l|}{ Yukon Bioswales } \\
\hline Nodding onion & Allium cernuum \\
\hline California aster & Symphyotrichum chilense \\
\hline Great camas & Camassia leichtlinii \\
\hline Beach daisy & Erigeron glaucus \\
\hline Spreading rush & Juncus patens \\
\hline Douglas aster & Aster subspicatus \\
\hline Idaho fescue & Festuca idahoensis \\
\hline Kinicknick & Arctostaphylos uva-ursi \\
\hline Dwarf coyote brush & Baccharis pilularis \\
\hline Manzanita & Arctostaphylos columbiana \\
\hline Showy milkweed & Asclepias speciosa \\
\hline Bluebells & Hyacinthoides \\
\hline Rough sedge & Carex muricata \\
\hline Tufted hairgrass & Deschampsia cespitosa \\
\hline California lilac & Ceanothus spp. \\
\hline Oregon iris & Iris tenax \\
\hline Douglas iris & Iris douglasiana \\
\hline Beach strawberry & Fragaria chiloensis \\
\hline Oregon sunshine & Eriophyllum lanatum \\
\hline Checkermallow spp. & Sidalcea spp. \\
\hline Penstemon spp. & Penstemon spp. \\
\hline Rose campion & Silene coronaria \\
\hline California poppy & Eschscholzia californica \\
\hline
\end{tabular}


Appendix A. List of plants that were initially planted by Green Lents at Johnson Creek Commons, Beyer Court Rain Garden and Yukon Bioswale enhanced pollinator sites.

\begin{tabular}{|l|l|}
\hline \multicolumn{2}{|c|}{ Beyer Court Rain Garden } \\
\hline Nodding onion & Allium cernuum \\
\hline Oregon grape & Mahonia aquifolium \\
\hline Great camas & Camassia leichtlinii \\
\hline Beach daisy & Erigeron glaucus \\
\hline Spreading rush & Juncus patens \\
\hline Red osier dogwood & Cornus sericea \\
\hline Idaho fescue & Festuca idahoensis \\
\hline Cascara & Rhamnus purshiana \\
\hline Blanketflower & Gaillardia aristata \\
\hline Salal & Gaultheria shallon \\
\hline Oregon iris & Iris tenax \\
\hline Soft rush & Juncus effusus \\
\hline White meadowform & Limnanthes alba \\
\hline Twinberry honeysuckle & Lonicera involucrata \\
\hline Penstemon spp. & Penstemon spp. \\
\hline Pacific nine bark & Physocarpus capitatus \\
\hline Checkermallow spp. & Sidalcea spp. \\
\hline Oregon stone crop & Sedum oreganum \\
\hline Red flowering currant & Ribes sanguineum \\
\hline Western sword fern & Polystichum munitum \\
\hline Douglas aster & Aster subspicatus \\
\hline Idaho blue eyed grass & Sisyrinchium idahoense \\
\hline Rose spirea & Spiraea douglasii \\
\hline
\end{tabular}


Appendix B. Example of plant identification list that were provided to community scientists for monitoring bee pollinators floral associations.

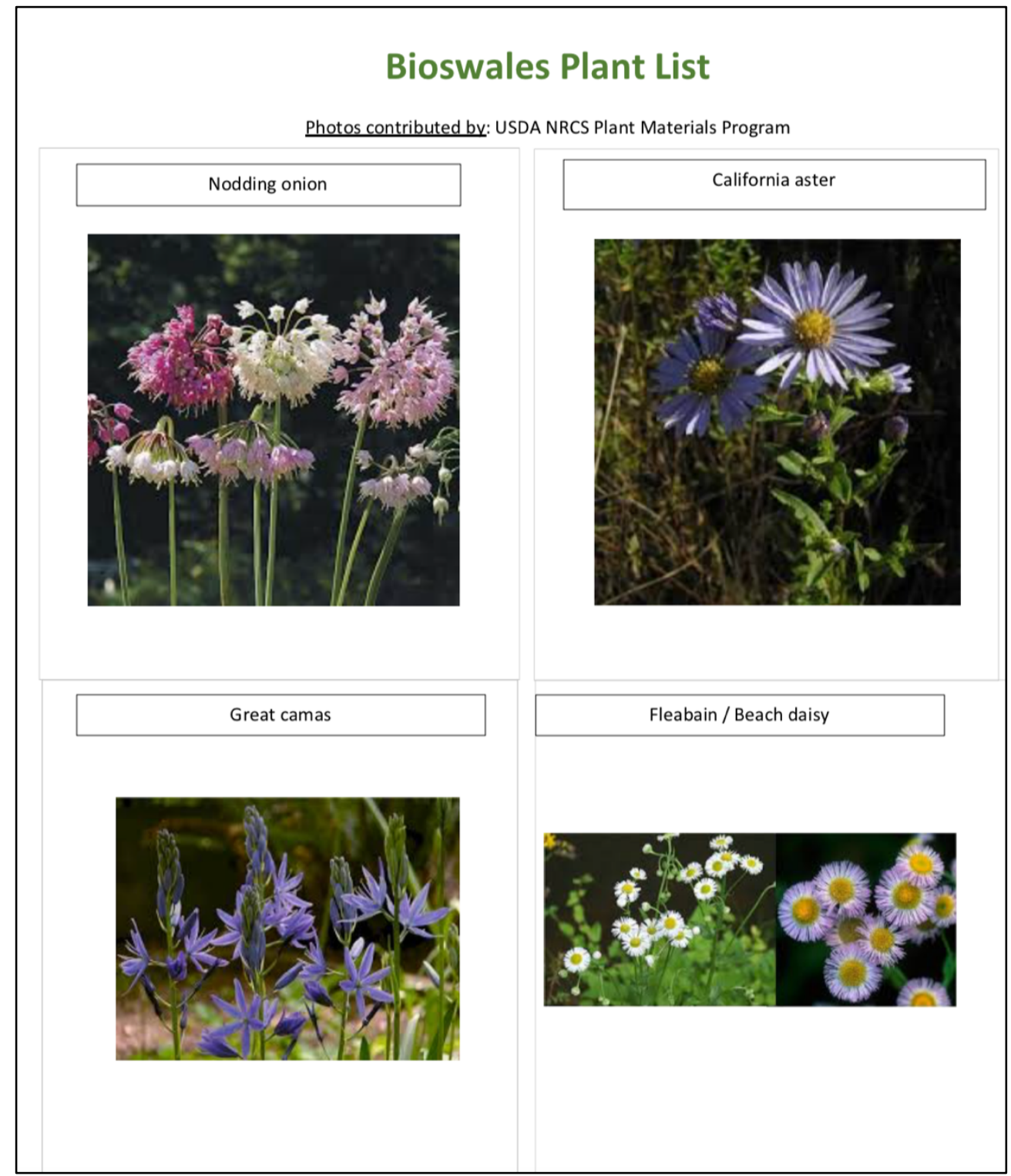




\section{Appendix C. Data collection sheet for bee observational data. Data collection sheet form from Minerath et al., (2014).}
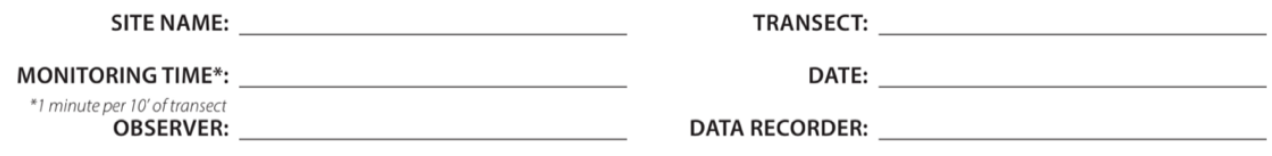

STEP 3-Site Conditions (Remember to note Observation End Time upon completion!)

OBSERVATION START TIME: SHADE TEMP: Wind: (circle one) Calm / light air / light breeze / gentle breeze OBSERVATION END TIME: ${ }^{\circ} \mathrm{C} /{ }^{\circ} \mathrm{F}$ Sky: (circle one) Clear / partly cloudy / bright overcast

\section{STEP 4-Monitoring}

Set timer and hit start when ready. Note any floral visitors you see and identify to your confidence level. Pace the transect until the time is up. FLORAL VISITOR CATEGORIES:

Bees:

Honey bee

Bumble bee

Chap leg bee

Medium dark bee

Metallic green bee

Striped sweat bee

Tiny dark bee

Striped hairy belly bee

Metallic hairy belly be

Cuckoo bee

Butterfly

$\begin{array}{ll}\text { Moth } & \text { Wasp } \\ \text { Beetle } & \text { Spider }\end{array}$

OBSERVATIONS:

True bug

Important: Remember to look at a diversity of flowers, stand so that you do not cast a shadow, and only ID floral visitors to the level at which you are confident in your identification. In order to track floral attractiveness, tally individual floral visitors from the same species by floral association.

\begin{tabular}{|l|l|l|l|l|}
\hline$\#$ & FLORAL VISITOR & DESCRIPTION (GENUS, COLOR, SIZE, ETC.) & \# OBSERVED (TALLY) & FLORAL ASSOCIATION \\
\hline 1 & & & & \\
\hline 2 & & & & \\
\hline 3 & & & & \\
\hline 4 & & & & \\
\hline 5 & & & & \\
\hline 6 & & & & \\
\hline 7 & & & & \\
\hline 8 & & & & \\
\hline 9 & & & & \\
\hline 10 & & & & \\
\hline 11 & & & & \\
\hline
\end{tabular}


Appendix D. Comma-separated values (CSV) spreadsheet titled "Vegetation Survey Data".

Data 1 File Type: .txt

Size: $6 \mathrm{~KB}$

Name: Vegetation Survey Data

Description: Spreadsheet containing all vegetation survey metrics measured in the field (percent cover, frequency, density, blossom density), and relative proportion values of floral resources that were calculated after data were collected. The spreadsheet is a comma-separated value (CSV) file. This document is titled, "Vegetation Survey Data" and can be opened with Microsoft Excel or Numbers. 
Appendix E. Comma-separated values (CSV) spreadsheet titled "Bee Observations and Floral Resources".

Data 2 File Type: .txt

Size: $34 \mathrm{~KB}$

Name: Bee Observations and Floral Resources

Description: Spreadsheet containing observational bee monitoring data collected from June to August 2018. This spreadsheet contains all bees observed, what floral resource they were seen on, as well as relative proportions of floral resources. Spreadsheet is a comma-separated value (CSV) file. This document is titled, "Bee observations and floral resources" and can be opened with Microsoft Excel or Numbers. 